\title{
Astrocytes Control the Development of the Migration-Promoting Vasculature Scaffold in the Postnatal Brain via VEGF Signaling
}

\author{
Lusine Bozoyan, ${ }^{1}$ Jivan Khlghatyan, ${ }^{1}$ and Armen Saghatelyan ${ }^{1,2}$ \\ ${ }^{1}$ Cellular Neurobiology Unit, Centre de Recherche Université Laval Robert-Giffard, Quebec City, Quebec, Canada G1J 2G3, and ²Departement of Psychiatry \\ and Neuroscience, Université Laval, Quebec City, Quebec, Canada G1K 7P4
}

\begin{abstract}
New neurons are constantly being generated in the postnatal subventricular zone. They have to migrate long distances via the rostral migratory stream (RMS) to reach their final destination in the olfactory bulb (OB). In adults, these neuronal precursors migrate in chains, ensheathed by astrocytic processes, and travel toward the OB along blood vessels (BVs) that topographically outline the RMS. The molecular and cellular mechanisms leading to the development of the RMS and the formation of the migration-promoting vasculature scaffold in the adult mice remain unclear. We now reveal that astrocytes orchestrate the formation and structural reorganization of the vasculature scaffold in the RMS and, during early developmental stages, the RMS contains only a few BVs oriented randomly with respect to the migrating neuroblasts. The first parallel BVs appeared at the outer border of the RMS, where vascular endothelial growth factor (VEGF)-expressing astrocytes are located. Gain-of-function and loss-of-function experiments revealed that astrocyte-derived VEGF plays a crucial role in the formation and growth of new BVs. Real-time videoimaging also showed that the migration of neuronal precursors in the developing RMS differs substantially from neuronal displacement in the adult migratory stream partially because of not yet fully developed vasculature scaffold. The downregulation of VEGF in vivo, specifically in the astrocytes of the developing RMS, affected the development of the vasculature scaffold and led to alterations in neuroblast migration. Altogether, our results demonstrate that astrocytes orchestrate the formation and growth of parallel BVs, crucial migration-promoting scaffolds in the adult migratory stream, via VEGF signaling.
\end{abstract}

\section{Introduction}

Neuronal precursors, which arise in the subventricular zone (SVZ), migrate to the olfactory bulb (OB) throughout the life span of animals (Kriegstein and Alvarez-Buylla, 2009). They first migrate tangentially through the rostral migratory stream (RMS), a long and convoluted pathway, and then, when they reach the $\mathrm{OB}$, turn to migrate radially to the various bulbar layers (Lledo and Saghatelyan, 2005). In the adult RMS, neuroblasts travel in chains ensheathed by astrocytic processes (Lois and Alvarez-Buylla, 1994), and their migration is under the control of various signaling molecules, such as polysialated neural cell adhesion molecule (PSA-NCAM) (Cremer et al., 1994; Hu et al.,

Received Nov. 2, 2011; revised Dec. 13, 2011; accepted Dec. 21, 2011.

Author contributions: A.S. designed research; L.B., J.K., and A.S. performed research; L.B., J.K., and A.S. analyzed data; L.B. and A.S. wrote the paper.

This work was supported by a Canadian Institutes of Health Research grant to A.S. L.B. and J.K. were partially supported by Le Fonds québécois de la recherche sur la nature et les technologies (FQRNT) and Université Laval fellowships, respectively. A.S. is a recipient of the Canada Research Chair in postnatal neurogenesis. We thank Dr. Marina Snapyan for performing fluorescent in situ hybridization and for establishing the cell cultures, as well as the members of the laboratory for helpful discussions. We also thank Dr. Akiyoshi Uemura (Riken Center for Developmental Biology, Kobe, Japan) for providing the VEGF plasmid and the Platform for Cellular Imaging of (entre de Recherche Université Laval Robert-Giffard for producing the lentiviruses and retroviruses.

Correspondence should be addressed to Armen Saghatelyan, Centre de Recherche Université Laval RobertGiffard, Université Laval, 2601 chemin de la Canardiere, Quebec City, Quebec, Canada G1J 2G3. E-mail: armen.saghatelyan@crulrg.ulaval.ca.

DOI:10.1523/JNEUROSCI.5531-11.2012

Copyright $\odot 2012$ the authors $\quad 0270-6474 / 12 / 321687-18 \$ 15.00 / 0$
1996), slits (Nguyen-Ba-Charvet et al., 2004), ephrins (Conover et al., 2000), integrins (Murase and Horwitz, 2002), ErbB4 (Anton et al., 2004), GABA (Bolteus and Bordey, 2004; Snapyan et al., 2009), and different growth factors such as GDNF (glial cell linederived neurotrophic factor) (Paratcha et al., 2006), brainderived neurotrophic factor (BDNF) (Chiaramello et al., 2007; Snapyan et al., 2009), and vascular endothelial growth factor (VEGF) (Wittko et al., 2009). In addition to these molecular cues, cellular interactions between neuroblasts, astrocytes, and blood vessels (BVs) are required for faithful migration toward the OB. Adult neuronal precursors use for their migration BVs that topographically outline the RMS (Snapyan et al., 2009; Whitman et al., 2009) and create a migratory path by repelling astrocytic processes (Kaneko et al., 2011). However, it is not known whether these molecular and cellular interactions play a role in guiding neuronal precursors into the developing $\mathrm{OB}$ and which mechanisms are involved in the development of the migratory pathway. The few studies that have addressed the development of the RMS have shown that chain formation of neuroblasts occurs late in postnatal development (Pencea and Luskin, 2003; Peretto et al., 2005), that astrocytes in early developmental stages are positioned at the outer border of the migratory stream (Law et al., 1999; Peretto et al., 2005), and that the RMS vasculature is laid down during postnatal development (Colín-Castelán et al., 2011). However, nothing is known about the mechanisms controlling the appearance and development of the migration- 
promoting vasculature scaffold or how various cellular elements of the migratory pathway interact to orchestrate the development and structural reorganization of the RMS. Furthermore, in light of the major structural differences between the developing and the adult RMS, an important issue to be resolved is how neuroblast migration occurs during the different developmental stages.

We showed that the profile of vasculature development in the RMS strikingly resembles the profile of the appearance of astrocytes in the migratory stream and that these glial cells regulate the development and structural rearrangement of the vasculature scaffold via VEGF signaling. The downregulation of VEGF in vivo, specifically in the astrocytes of the developing RMS, affects the vasculature scaffold development that, consequently, leads to the alteration in the neuronal migration and decreased number of BrdU + cells arriving into the OB. Furthermore, we also showed that neuroblast migration is less efficient in the developing RMS compared with the adult migratory stream.

\section{Materials and Methods}

Animals. C57BL/6 mice (3, 7, 14, 21, and $60 \mathrm{~d}$ old; Charles River) and GAD67-green fluorescent protein (GFP) (Ono et al., 2005) and glial fibrillary acidic protein (GFAP)-GFP (The Jackson Laboratory) transgenic mice were used. Animals of either sex were used for 3- to 21-d-old mice, and only males were used for the adult animals. All the animal experiments were approved by the animal protection committee of Université Laval. The mice were kept on a $12 \mathrm{~h}$ light/dark cycle at a constant temperature $\left(22^{\circ} \mathrm{C}\right)$ with food and water ad libitum.

Immunohistochemistry and BrdU labeling. Immunohistochemistry procedures were performed as described previously (Snapyan et al., 2009). The mice were deeply anesthetized and transcardially perfused with $0.9 \% \mathrm{NaCl}$ followed by $4 \%$ paraformaldehyde (PFA). The brains were postfixed in $4 \%$ PFA overnight at $4^{\circ} \mathrm{C}$. Immunohistochemistry was performed on $40-\mu \mathrm{m}$-thick free-floating vibratome sections. The following primary antibodies were used: rabbit anti-VEGF-A (1:200; Santa Cruz Biotechnology), guinea pig (1:1000; Abcam) and goat (1:1000; Santa Cruz Biotechnology) anti-doublecortin (Dcx), mouse anti-PSANCAM (1:1000; Millipore), mouse (1:200; Serotec) and rat (1:200; Serotec) anti-5-bromo-2+-deoxyuridine (BrdU), rabbit (1:1000; Dako) and mouse (1:1000; Millipore) anti-GFAP, rabbit anti-Ki67 (1:1000; Abcam), rat anti-platelet/endothelial cell adhesion molecule (PECAM) (1:100; BD Pharmingen), rabbit anti-brain lipid-binding protein (BLBP) (1:300; Millipore), rabbit anti-ZsGreen (1:500; Clontech), and rabbit (1:1000; Invitrogen) and mouse (1:1000; Invitrogen) anti-GFP. Corresponding secondary antibodies were used.

The DNA synthesis marker BrdU (Sigma-Aldrich) was dissolved in a sterile solution of $0.9 \% \mathrm{NaCl}$ and $1.75 \% \mathrm{NaOH}(0.4 \mathrm{~N})$. This solution was injected intraperitoneally at a concentration of $50 \mathrm{or} 10 \mathrm{mg} / \mathrm{kg}$ body weight. A single-shot BrdU injection $(50 \mathrm{mg} / \mathrm{kg}$ ) was performed $1 \mathrm{~h}$ before the animals were killed to visualize BrdU+ cells in the postnatal RMS. To evaluate the number of newborn cells arriving into the $\mathrm{OB}$, a single shot of $\mathrm{BrdU}(10 \mathrm{mg} / \mathrm{kg})$ was performed at postnatal day 12 (P12) and animals were killed at P19. Before performing the BrdU immunohistochemistry, the slices were treated with $2 \mathrm{~N} \mathrm{HCl}$ for $45 \mathrm{~min}$ at $37^{\circ} \mathrm{C}$. Functional BVs were visualized by injecting fluorescent tracers dextran Texas Red or Cascade Blue into the hearts of the animals. Five minutes later, the brains were removed and fixed in $4 \% \mathrm{PFA}$ for $24 \mathrm{~h}$ at $4^{\circ} \mathrm{C}$. Three different molecular-weight fluorescent tracers were used (3000 and $70,000 \mathrm{kDa}$ dextran Texas Red and 10,000 kDa Cascade Blue; Invitrogen). No difference in the quantity or intensity of BV labeling was observed. We distinguished the RMS by DAPI (Invitrogen) nuclear counterstaining, according to the manufacturer's instructions. Images were acquired with an FV1000 confocal microscope equipped with UV (405 nm), argon (488 nm), helium-neon (543 nm), and helium-neon $(633 \mathrm{~nm})$ lasers (Olympus). Double- and triple-immunolabeled cells were analyzed using three-dimensional (3D) reconstructed images with $x-z$ and $y-z$ orthogonal projections. Image analysis was performed manually using Fluoview software. The area of the RMS was calculated based on DAPI staining, with $10-15 \%$ of the width of the RMS defined as the RMS border. BV length was calculated based on PECAM immunostaining. BV density was calculated by normalizing the length of the BVs to the volume of the RMS. BVs were considered parallel if they were forming an angle of $<10^{\circ}$ with the line outlining the RMS (range, $0.2-8^{\circ}$; mean, $3 \pm$ $0.4^{\circ}$ ). The angle was measured using an ImagePro software (MediaCybernetics) and represents the angle from the line drawn on the blood vessel to the second line outlining the RMS, in a clockwise direction. BVs forming an angle of $>10^{\circ}$ with the line outlining RMS were considered nonparallel (range, $11.5-76^{\circ}$; mean, $36.6 \pm 3.6^{\circ}$ ). As another measure for the presence of parallel BVs, we also calculated the number of BV branching points normalized to the density of the BVs. BV functionality was assessed by colocalization of the PECAM endothelial marker and the dextran Texas Red tracer. Assuming that the tracer does not penetrate into nonfunctional BVs, PECAM+/dextran + BVs were considered functional and PECAM +/dextran - BVs nonfunctional. The number of nonfunctional BVs was calculated as a percentage of total PECAM+BVs. The volume of the entire RMS, from the OB until SVZ, was calculated on each third serially prepared DAPI-counterstained $40-\mu$ m-thick section using the following equation:

$$
\sum_{i=0}^{n} A_{i} d
$$

where $A$ is the area of the $i$ th section, $d$ is the distance between analyzed sections, and $n$ is the total number of measured sections.

In situ hybridization. For the in situ hybridization studies, antisense RNA probes were obtained from the VEGF plasmid (kindly provided by Dr. A. Uemura, Riken Center for Developmental Biology, Kobe, Japan) and were purified using ProbeQuant G-50 columns (GE Healthcare). Antisense RNA probes were labeled using DIG RNA labeling kits (1:2000; Roche Diagnostics). In situ hybridization was performed on $40-\mu \mathrm{m}-$ thick free-floating vibratome sections. The sections were prehybridized in hybridization solution (50\% formamide, $4 \%$ SSC, $0.05 \%$ Denhardt, $0.05 \%$ salmon sperm DNA, $0.025 \%$ tRNA) for $1 \mathrm{~h}$ at $45^{\circ} \mathrm{C}$ and hybridized overnight with a DIG-labeled VEGF antisense probe at $45^{\circ} \mathrm{C}$. The sections were then incubated with anti-DIG antibody (Roche Diagnostics) overnight at $4^{\circ} \mathrm{C}$. The signals were revealed using nitroblue-tetrazoliumchloride/5-bromo-4-chlor-indolyl-phosphate (Promega). For fluorescent in situ hybridization, anti-DIG-horse-radish peroxidase POD antibodies were used, and the signal was amplified with the TSH-Plus tetramethylrhodamine System (PerkinElmer Life and Analytical Sciences) following the manufacturer's instructions.

Primary astrocyte cultures and immunocytochemistry. To prepare astrocyte cultures from P3 and P60 SVZ, P3 cortex, and P3 cerebellum, these regions were dissected out under a microscope and immersed in HBSS containing $10 \mathrm{~mm}$ HEPES. The isolated brain tissue was then triturated into a single-cell suspension and resuspended in $10 \mathrm{ml}$ of growth medium. Cells were cultured under adherent conditions in the presence of $10 \%$ fetal bovine serum, $5 \%$ horse serum, and DMEM/F-12 supplemented with B27 and growth factors ( $20 \mathrm{ng} / \mathrm{ml} \mathrm{EGF}$ and $10 \mathrm{ng} / \mathrm{ml} \mathrm{FGF2}$ ). The medium was changed every second day.

For the immunocytochemistry procedures, the cultures were rinsed with PBS (three times for $5 \mathrm{~min}$ ), fixed in 4\% PFA (40 min), washed in PBS (three times for $5 \mathrm{~min}$ ), permeabilized in $0.2 \%$ Triton X-100 ( $2 \mathrm{~h}$ at room temperature), and incubated overnight with primary antibodies in $0.2 \%$ Triton X-100-4\% BSA at $4^{\circ} \mathrm{C}$. The cultures were then rinsed with PBS (three times for $10 \mathrm{~min}$ ), incubated with secondary antibodies for $3 \mathrm{~h}$ at room temperature, rinsed with PBS (three times for $10 \mathrm{~min}$ ), counterstained with DAPI, and mounted in fluorescent mounting medium (Dako).

$3 D$ aortic ring cultures. Dorsal aortas were dissected from deeply anesthetized 2- to 3-month-old male C57BL/6 mice the day after the preparation of the astrocyte cultures, except that to compare the angiogenic effects of the conditioning medium of $\mathrm{P} 3$ versus adult $\mathrm{SVZ}$ astrocytes, the dorsal aortas were dissected on the sixth day after the preparation of the astrocyte cultures because of lower cell density of adult SVZ astrocytes after $2 \mathrm{~d}$ in cultures. The aortas were dissected in ice-cold HBSS contain- 
ing $10 \mathrm{~mm}$ HEPES and cross-sectioned into 1-mm-thick rings under a dissecting microscope. The aortic rings were placed on Matrigel-coated coverslips in 24-well dishes and covered with an additional layer of Matrigel, creating a 3D structure that allowed the growth of the BVs. To compare the angiogenic properties of astrocytes at different ages and from different brain regions, the conditioning medium derived from primary cultures of P3 and adult SVZ, P3 cortex, and P3 cerebellum astrocytes was added to the aortic rings every second day. The cultures were analyzed $7 \mathrm{~d}$ later using an FV1000 confocal microscope (Olympus). The BVs were visualized in bright-field images based on their long tubular structure and identified by post hoc staining with PECAM antibodies. The length of the BVs that received astrocyte conditioning medium (Ast. CM) from the various brain regions was measured using the Fluoview program and compared with the length of control BVs (that received unconditioned culture medium).

Exogenous VEGF application, siRNA transfection, and miRNA preparation. To understand the role of VEGF in BV growth and development, exogenous VEGF (PeproTech) was added to the culture medium of the aortic rings at final concentrations of 2, 10, 25, 50, and $100 \mathrm{ng} / \mathrm{ml}$. The culture medium was changed every $48 \mathrm{~h}$. The lengths of the test BVs (with VEGF) were measured after $7 \mathrm{~d}$ and compared with the lengths of the control BVs (without VEGF).

Astrocyte transfections were performed using HiPerFect transfection reagent (QIAGEN) according to the manufacturer's instructions. VEGF and control siRNAs were from Santa Cruz Biotechnology (sc-36815 and sc-37007, respectively). VEGF siRNAs were a pool of three target-specific 20-25 nt siRNA designed to knock down VEGF gene expression. Nontargeting siRNA was used as a negative control. The transfections were performed using $500 \mathrm{ng}$ of siRNA. Total RNA was prepared using Invitrogen TRIzol reagent according to the manufacturer's instructions. siRNA efficiency was verified by PCR using the sc-36815-PR primer (Santa Cruz Biotechnology). To investigate the effect of VEGF knockdown in P3 SVZ astrocytes on the growth of BVs, three astrocyte cultures per experiment were prepared at $48 \mathrm{~h}$ intervals. The astrocyte cultures were transfected on day 3 , and their conditioning medium was added to the aortic ring cultures ( $2 \mathrm{~d}$ after transfection). The conditioning medium from subsequently transfected astrocyte cultures was added to the aortic ring cultures every $48 \mathrm{~h}$. The aortic rings were fixed and analyzed after $7 \mathrm{~d}$ of culture.

Anti-VEGF miRNA was produced using BLOCK-iT miR RNAi Select kits (Invitrogen). Briefly, dsoligos were generated based on the manufacturer's instructions and ligated to the pcDNA6.2-GW/EmGFP-miR vector. The expressing cassette, which included the Emerald-GFP (EmGFP) and the pre-miRNA sequence, was then introduced into the blunted-SacI and SacII sites of a pHAGE-modified lentiviral vector (a kind gift from R. C. Mulligan, Harvard Medical School, Boston, MA). It is noteworthy that the human cytomegalovirus early promoter had previously been replaced by the GFAP promoter from the original pHAGE vector. We generated four anti-VEGF miRNA expression vectors based on four forward and four reverse oligos provided by manufacturer. Two of four anti-VEGF miRNA expression vectors induced downregulation of VEGF in astrocyte cultures. These expression vectors were used for lentivirus production (VEGF miRNA 10 and VEGF miRNA 11). Cotransfection of the backbone vector, including the expression cassette and helper plasmids [encoding the viral genes Gag-Pol, Tat, Rev, and vesicular stomatitis virus (VSV-G)] was done into 293 T cells. The cell supernatant was centrifuged $(2 \mathrm{~h}, 48,950 \times \mathrm{g})$, aliquoted, and stored at $-80^{\circ} \mathrm{C}$ until injected. We used BLOCK-iT miR-lacZ-validated miRNA (Invitrogen) as a control vector and produced lentivirus with the GFAP promoter. We also used lentivirus expressing ZsGreen protein under GFAP promoter to ascertain the specificity of astrocytic targeting.

Coculture of RMS explants with control or VEGF miRNA-infected astrocytes. To assess whether VEGF downregulation in astrocytes affects the astrocytic properties that, consequently, might alter the neuronal migration, we cocultured RMS explants with control or VEGF miRNAinfected astrocytes. To infect the astrocytes with control and VEGF miRNAs, the freshly dissociated SVZ cell suspension was left with the virus for $1 \mathrm{~h}$ at $37^{\circ} \mathrm{C}$ and was shaken every $15 \mathrm{~min}$. The efficiency of infection was $61.4 \pm 1.8 \%$. The cells were placed on Matrigel-coated coverslips, and $72 \mathrm{~h}$ later, RMS explants were placed on the infected astrocytes, allowing the direct contact between astrocytes and explants. RMS explants were excised from $250-\mu \mathrm{m}$ thick P4-P5 acute slices as described previously (Saghatelyan et al., 2004). To allow the growth of explants in three dimensions, the RMS explants were covered with another layer of Matrigel. The explants were analyzed 24-36 h later. The length of neuroblast chains formed from the RMS explants was measured using the Fluoview program, and the mean length was compared between explants cocultured with either control or VEGF miRNA-infected astrocytes.

Stereotaxic injections. To label newborn neurons, a GFP-encoding retrovirus was injected into the SVZ for analysis of cell migration in the RMS as well as for calculating the distribution of neuroblast distance from BVs in the RMS center and borders. Injections were performed in 2-, 10-, and 60-d-old C57BL/6 mice at the following coordinates (in mm): for P2 (with respect to the $\mathrm{BV}$ intersection on lambda): anteroposterior, 2.2; mediolateral, 1.2; dorsoventral, 1.5; for P10 (with respect to the bregma): anteroposterior, 1.2; mediolateral, 0.7; dorsoventral, 1.75; for P60 (with respect to the bregma): anteroposterior, 0.7 ; mediolateral, 1.2 ; dorsoventral, 1.9. To downregulate VEGF expression in astrocytes during the early stages of postnatal development, EmGFP-miVEGF and control EmGFPmiLacZ lentiviral vectors controlled by the GFAP promoter were injected at two sites in the RMS of P4 pups at the following coordinates (in millimeters with respect to the BV intersection on lambda): to target the horizontal limbs of the RMS: anteroposterior, 5.6; mediolateral, 0.62 ; dorsoventral, 2.5; to target the vertical limbs of the RMS: anteroposterior, 5.0; mediolateral: 0.62; and dorsoventral: 2.2. The pups were killed at $\mathrm{P} 14$, and BV density and organization were analyzed using an FV1000 confocal microscope (Olympus). To evaluate the dynamics of neuroblast migration at P14 after VEGF downregulation in astrocytes, a mCherryexpressing lentivirus was injected in the SVZ at P10.

To calculate the shortest distance of neuroblasts from BVs filled with the fluorescent tracer dextran Texas Red or Cascade Blue in wild types, as well as in control and VEGF miRNA-injected mice, GFP-expressing retroviruses were injected into the SVZ, and analysis was performed in the RMS. To evaluate the effect of vasculature scaffold alterations on the migration of newborn cells after astrocyte-specific downregulation of VEGF, we injected BrdU $(10 \mathrm{mg} / \mathrm{kg})$ at P12 in animals that were previously injected with control or VEGF miRNA at P4. We killed animals at $\mathrm{P} 19$ and calculated the density of BrdU + cells in the granule cell layer (GCL) of OB, RMS, and in the regions neighboring RMS (200- $\mu \mathrm{m}$-width area outside of horizontal limb of the RMS).

Slice preparation and time-lapse videoimaging. Sagittal sections (300 and $250 \mu \mathrm{m}$ thick) from young (P5 and P14) and adult (2-3 months old) mouse forebrains, respectively, were prepared and maintained at $32^{\circ} \mathrm{C}$. The slices were constantly perfused $(2 \mathrm{ml} / \mathrm{min})$ with artificial CSF (ACSF) containing the following (in mM): $125 \mathrm{NaCl}, 26 \mathrm{NaHCO}_{3}, 10$ glucose, $3 \mathrm{KCl}, 2 \mathrm{CaCl}_{2}, 1.3 \mathrm{MgCl}_{2}$, and $1.25 \mathrm{NaH}_{2} \mathrm{PO}_{4}$ (bubbled with $95 \% \mathrm{O}_{2} / 5 \% \mathrm{CO}_{2}$ ), $\mathrm{pH}$ 7.4. Multiple z-stack images (at least 6-10 $z$-sections, at $3 \mu \mathrm{m}$ intervals) were acquired every $30 \mathrm{~s}$ for $2 \mathrm{~h}$ with a BX61WI (Olympus) upright microscope equipped with a CCD camera (CoolSnap HQ2). Migrating cells were analyzed in three dimensions using the Imaris program (Bitplane). Multiple $z$-step acquisition in our time-lapse experiments of cell migration allowed us to follow the same cell in different $z$ plans. To define a stationary phase, we set an automatic threshold corresponding to the mean of SDs of cells displacement per second for all cells multiplied 1.5 times. This threshold was $0.025 \mu \mathrm{m} / \mathrm{s}$ for all analyzed groups, except for P5 where the threshold was 0.013 $\mu \mathrm{m} / \mathrm{s}$. The accuracy of the threshold was confirmed by visual inspection of stationary and migrating phases of migrating neuroblasts. For timelapse videoimaging of $\mathrm{mCherry}^{+}$neuroblasts after in vivo VEGF downregulation, the imaging was performed in the RMS regions containing $\mathrm{GFP}+$ astrocytes.

Western blot analysis. Acute sagittal slices of mouse forebrains at different developmental stages were prepared from GFAP-GFP animals as described. The slices were transferred to a fluorescent dissecting microscope (Olympus), and the RMS was carefully dissected $(100 \times$ magnification). For experiments with in vivo VEGF downregulation, only the areas of the RMS containing GFP+ labels were dissected. The tissue was 
homogenized in lysis buffer $(50 \mathrm{~mm} \mathrm{HCl}, \mathrm{pH}$ 7.5, 1 mm EDTA, $1 \mathrm{~mm}$ EGTA, $1 \mathrm{~mm}$ sodium orthovanadate, $50 \mathrm{~mm}$ sodium fluoride, $5 \mathrm{~mm}$ sodium pyrophosphate, $10 \mathrm{~mm}$ sodium $\beta$-glycerophosphate, $0.1 \%$ 2-mercaptoethanol, 1\% Triton X-100) containing Protease Inhibitor Cocktail Set III (Calbiochem). The homogenates were sonicated and centrifuged at $13,000 \times g$ at $4^{\circ} \mathrm{C}$ for $20 \mathrm{~min}$ to remove insoluble material. To determine whether the astrocytes secreted VEGF, the conditioning medium of astrocyte cultures was collected from 5 to $7 \mathrm{~d}$ cultures, and proteins were precipitated using acetone. Five volumes of precooled acetone $\left(-30^{\circ} \mathrm{C}\right)$ were added to $1 \mathrm{vol}$ of conditioning medium. The mixture was incubated overnight at $-30^{\circ} \mathrm{C}$. Proteins were isolated by centrifugation at $10,000 \mathrm{rpm}$ at $4^{\circ} \mathrm{C}$ for $15 \mathrm{~min}$ and were resuspended in $1 \times$ RIPA buffer. The total protein concentration was determined using the Bradford assay (Bio-Rad). Protein samples were separated on $\mathrm{Nu}-$ Page 4-12\% Bis-Tris gels (Invitrogen) and transferred to nitrocellulose membranes (GE Healthcare Bio-sciences). The following primary antibodies were used: rabbit anti-VEGF (1:1000; Abcam), mouse anti-GFAP (1:1000; Millipore), and mouse anti-actin (1:3000; Cedarlane). Goat anti-rabbit IgG HRP (1:3000; Millipore) and goat anti-mouse HRP (1: 3000; Bio-Rad) secondary antibodies and an ECL chemiluminescence substrate mixture (GE Healthcare) were used to visualize the bands. For the miRNA experiments, we also immunoblotted for GFP (1:1000; Invitrogen) to verify the presence of infected cells.

Statistical analysis. Results are expressed as means \pm SEM. Statistical significance was determined by an ANOVA for multiple groups and a Student's $t$ test to compare two groups $\left({ }^{\star} p<0.05,{ }^{* *} p<0.001\right.$, and $\left.{ }^{* * *} p<0.0001\right)$.

\section{Results}

The vasculature scaffold is first laid down at the outer border of the developing RMS

To characterize the development of the RMS, we performed a detailed morphological analysis of the migratory pathway during different developmental stages. Brains were obtained from P3, P7, P14, P21, and adult mice. The RMS was identified by its high cellular density in DAPI-counterstained slices (Fig. 1A). In agreement with previous reports (Law et al., 1999; Peretto et al., 2005), we found that the RMS was wider during early developmental stages and subsequently decreased until adulthood. The width of the RMS at the horizontal limb, the largest part of the migratory stream, was $273.6 \pm 6.56,233 \pm 5.72,211.8 \pm 3.88,171.97 \pm$ 5.47, and $133 \pm 7.81 \mu \mathrm{m}$ for P3, P7, P14, P21, and P60, respectively ( $p<0.05$ between groups; $n=5-6$ animals per group). Interestingly, however, when the total volume of the RMS from the OB to the SVZ was calculated on serially prepared coronal sections, no difference with respect to developmental stage was observed (Fig. 1 B; P3, $2.32 \pm 0.18$; P7, $2.22 \pm 0.27$; P14, $1.98 \pm$ 0.34; P21, $2.08 \pm 0.15$; adult, $1.99 \pm 0.095 ; \times 10^{8} \mu \mathrm{m}^{3} ; n=3$ animals per group). These results suggested that, because of brain growth, the RMS becomes longer and thinner and that its total volume remains stable during postnatal development.

To investigate the organization of BVs during RMS postnatal development, we labeled BVs in P3, P7, P14, P21, and adult mouse brains using an antibody directed against PECAM, an endothelial cell marker. During the first postnatal week, there were only a few BVs in the RMS, which were organized randomly with respect to the migrating neuroblasts (Fig. $1 A$ ). Unlike the neighboring striatum where the density of BVs stabilized at the first to second postnatal weeks (data not shown), the density of the BVs in the RMS increased throughout postnatal development (Fig. $1 C$; P3, $1.07 \pm 0.08 ; \mathrm{P} 7,1.05 \pm 0.06 ; \mathrm{P} 14,1.3 \pm 0.06 ; \mathrm{P} 21$, $1.6 \pm 0.1$; adult, $1.7 \pm 0.2 ; \times 10^{3}$ per cubed millimeter; $p<0.05$; $n=4-6$ animals per group; $p<0.05$ from P3-P7 until adulthood). Interestingly, however, whereas the overall density of BVs during early developmental stages was lower, the density of BVs at the outer border of the RMS (calculated as $10-15 \%$ of the width of the RMS) was consistently higher than inside the migratory stream for the same developmental period (data not shown). Since we previously showed that adult neuronal precursors migrate toward the OB along the parallel BVs (Snapyan et al., 2009), we calculated the density of the parallel BVs at the outer border of and inside the RMS at different developmental stages. As with the overall density of BVs, the density of the parallel BVs increased in the RMS as development proceeded, and the border contained consistently more parallel BVs than the core of the RMS during early developmental stages (Fig. $1 D$; P3 RMS, $0.33 \pm 0.05$ vs P3 border, $1.15 \pm 0.13$; P7 RMS, $0.27 \pm 0.03$ vs P7 border, $1.03 \pm$ 0.11; P14 RMS, $0.45 \pm 0.05$ vs P14 border, $1.10 \pm 0.08$; P21 RMS, $1.00 \pm 0.08$ vs $\mathrm{P} 21$ border, $0.94 \pm 0.07$; adult RMS, $1.39 \pm$ $0.11 ; \times 10^{3}$ per cubed millimeter; $n=4-6$ animals per group; $p<0.05$ for P3, P7, and P14 with a paired $t$ test). As another measure to reveal the presence of parallel blood vessels in the RMS, we calculated the number of branching points of BVs and normalized it to the density of BVs. The regions containing a higher density of parallel BVs will show a lower number of branching points normalized to the BV density. Indeed, the number of branching points of BVs per total BV density at the outer border of the developing RMS was much lower compared with the core of RMS (Fig. 1 E; P3 RMS, $12.5 \pm 0.5$ vs P3 border, $3.8 \pm$ 0.6; P7 RMS, $10.1 \pm 1.6$ vs P7 border, $3.5 \pm 0.6$; P14 RMS, $7.4 \pm$ 0.3 vs P14 border, $2.1 \pm 0.2$; P21 RMS, $4.7 \pm 0.5$ vs P2 1 border, $2.0 \pm 0.2$; adult RMS, $3.6 \pm 0.7 ; \times 10^{3}$ per density of BVs; $n=$ $4-6$ animals per group; $p<0.05$ for P3, P7, P14, and P21 with a paired $t$ test). These results suggested that, during early developmental stages, the RMS contained two distinct subdomains characterized by different overall BV densities, parallel BV densities, and BV branching. The presence of proliferating BrdU+/PE$\mathrm{CAM}+($ Fig. $1 F$ ) and migrating (also known as tip cells) (Fig. $1 G$ ) endothelial cells during early developmental stages, but not in adulthood, provided further support for these results (Fig. $1 F$ and data not shown). Interestingly, the presence of proliferating and migrating endothelial cells in the developing RMS was restricted to the outer border of the migratory stream, the RMS subregion where the first parallel BVs also appeared. Altogether, these results suggested that the migration-promoting vasculature scaffold is first laid down at the border of the developing RMS. During later developmental stages (P14 and P21), the RMS underwent continuous reorganization and began to resemble the adult migratory stream. During these stages, the density and overall length of parallel BVs increased (Fig. $1 C, D$ ). In addition, during late developmental stages, the percentage of nonfunctional BVs, i.e., the percentage of endothelial cell marker PECAM+-expressing BVs that did not colocalize with the dextran Texas Red tracer injected into the blood stream, decreased (Fig. $1 \mathrm{H}, \mathrm{I}$; P3 RMS, $10.0 \pm 1.3 \%$ vs P3 border, $11.3 \pm 1.3 \%$; P7 RMS, $7.4 \pm 0.8 \%$ vs P7 border, $8.9 \pm 1.3 \%$; P14 RMS, $2.1 \pm 0.3 \%$ vs P14 border, $2.0 \pm 0.4 \%$; P21 RMS, $0.5 \pm 0.2 \%$ vs P21 border, $0 \%$; adult RMS, $0.03 \pm 0.03 \% ; n=5-6$ animals per group). These results indicated that there is a major structural reorganization in the RMS during postnatal development leading to the appearance of parallel BVs that are a crucial migrationpromoting scaffold in adulthood. Our data also suggested that the vasculature scaffold is first laid down at the outer border of the migratory stream. These findings raise an important question about the molecular mechanisms regulating the formation of the vasculature scaffold in the RMS. 
P3

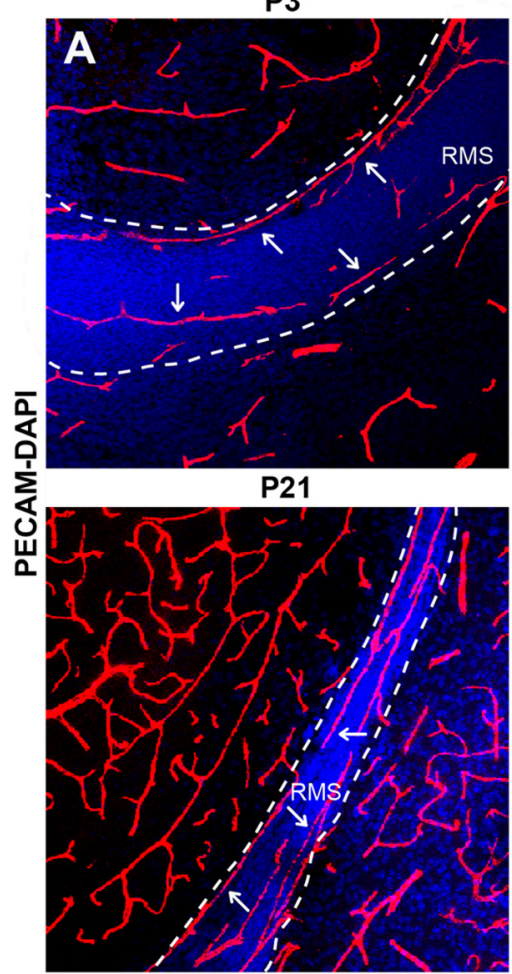

C

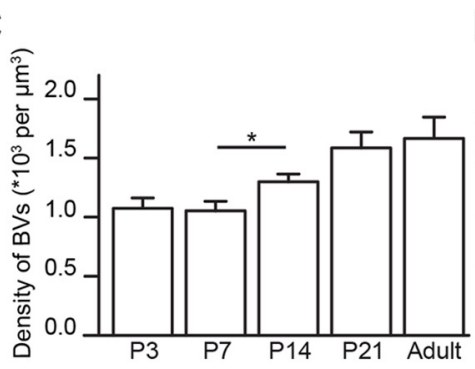

P3

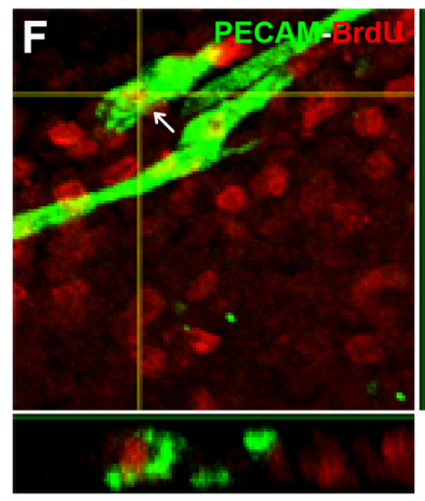

P3

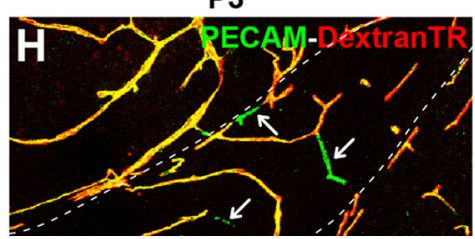

P7

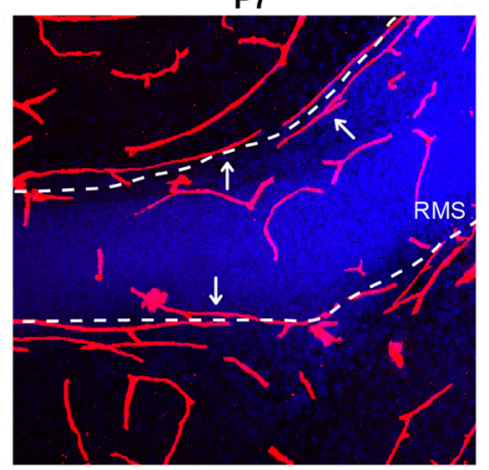

P60

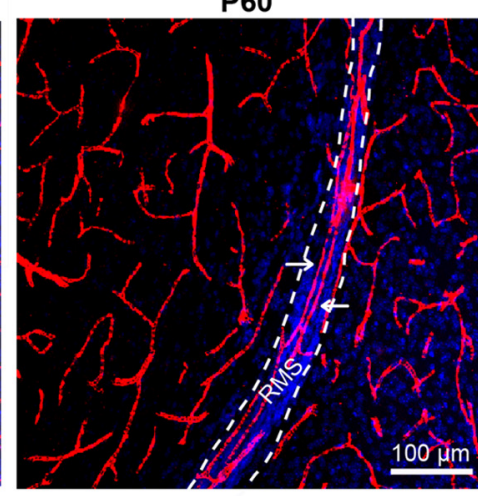

D

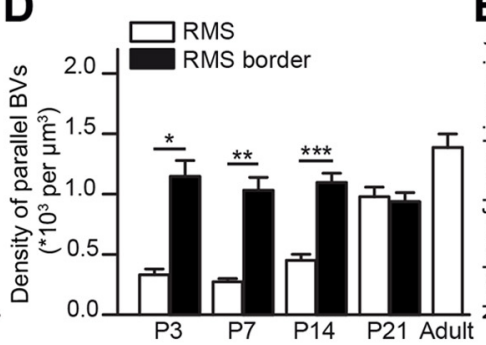

P60
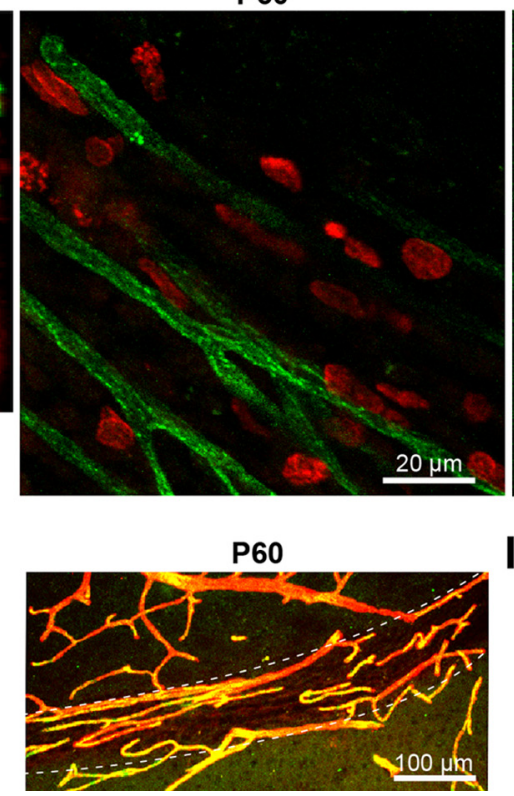

P14

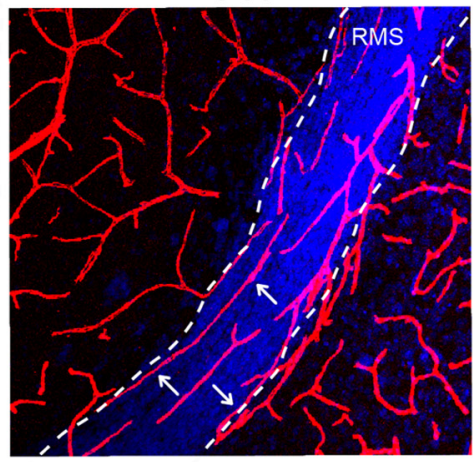

B
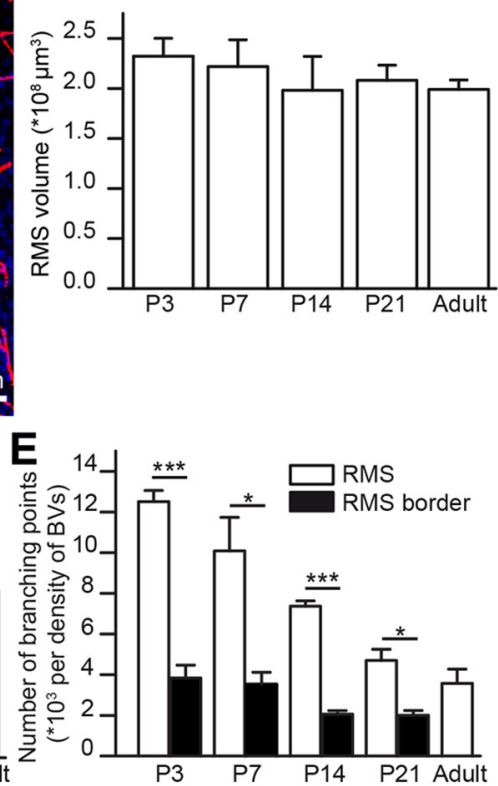

P3
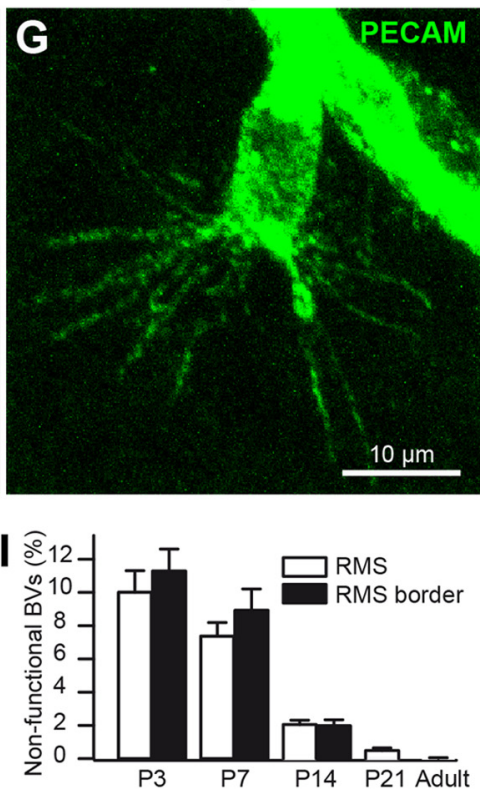

Figure 1. Development of vasculature scaffold in the RMS. A, Micrographs displaying BVs in the RMS at different developmental periods. Blood vessels were labeled by PECAM immunostaining, and RMS was visualized by dense DAPI labeling. Note that the first parallel BVs (arrows) appear at the outer border of the RMS. Note also that RMS width is decreasing from P3 to P60. RMS is indicated by the dashed white line. $\boldsymbol{B}$, Quantification of the total RMS volume, from the OB until SVZ, at different developmental periods. $(-\boldsymbol{E}$, Quantification of BVs density (C), the density of parallel BVs (D) and the number of $\mathrm{BV}$ branching points normalized to the density of BVs $(\boldsymbol{E})$ at different developmental periods. At early developmental periods, the parallel BVs are longer at (Figure legend continues.) 
The profile of vasculature development in the RMS resembles the profile of astrocyte appearance in the migratory stream

It is now well established that, during the first postnatal week, the radial glia undergoes a transformation leading to the appearance of astrocytes in the SVZ-RMS pathway (Alves et al., 2002). Astrocytes are first found at the outer border of the migratory stream and surround the masses of neuroblasts inside the RMS (Law et al., 1999; Pencea and Luskin, 2003; Peretto et al., 2005). Our results, which were in agreement with this, showed that, during early developmental stages, astrocytes identified by GFAP immunolabeling are mostly located at the outer border of the RMS (Fig. 2A), the region that harbors large numbers of proliferating and migrating endothelial cells and where the first parallel BVs appear (Fig. 1). The same results were obtained using GFAP-GFP mice (Fig. 3D) and BLBP immunostaining (data not shown).

Since it has been suggested that astrocytes may control vasculature development in the retina (Stone et al., 1995), we hypothesized that these glial cells may also guide vasculature formation in the RMS. To provide support for this hypothesis, we developed an in vitro method to test the role of astrocyte-derived molecular cues in the formation and growth of BVs. Astrocyte cultures were first prepared from different regions of $\mathrm{P} 3$ brains such as the SVZ (including the anterior portion with the RMS), cortex, and cerebellum, as well as from the adult SVZ (Fig. $2 B$ ). The conditioning medium derived from these cultures was added to separately prepared 3D BV cultures (aorta rings in Matrigel) every $48 \mathrm{~h}$ for $7 \mathrm{~d}$ (Fig. $2 \mathrm{~B}$ ). This in vitro method allowed us to explore the role of glial cell-derived secreted factors on the growth and formation of BVs without any confounding effects from other cellular populations such as neuroblasts (Fig. $2 C, D$ ). These experiments revealed that only conditioning medium derived from P3 SVZRMS astrocytes potentiated the formation and growth of new BVs (Fig. 2 E, F; P3 cortex, $101.1 \pm 4.6 \%$; P3 cerebellum, $142.1 \pm$ 20.0\%; P3 SVZ-RMS, $206.3 \pm 21.5 \%$; adult SVZ-RMS, $109.6 \pm$ $15.2 \%$ compared with the control $100 \% ; n=6$ cultures from 4 independent experiments; $p<0.0001$ between P3 SVZ and control, P3 cortex, or adult SVZ cultures and $p<0.05$ between SVZ and cerebellum cultures, analyzed using the Student's $t$ test). To rule out the possibility that this effect was caused by differences in the purity or cell density of the astrocyte cultures derived from different brain regions, we immunostained the cultures with GFAP and counterstained them with DAPI. The colocalization of the astrocytic marker GFAP with nuclear DAPI showed that our cultures were very pure (Fig. $2 D$; cortex, $97.9 \pm 0.5 \%$; cerebellum, $98.8 \pm 0.4 \%$; SVZ-RMS, $99.0 \pm 0.3 \% ; n=3$ cultures). In

\section{$\leftarrow$}

(Figure legend continued.) the outer border of the RMS. $\boldsymbol{F}$, Proliferating endothelial cells in the RMS at the beginning of postnatal development. Proliferating endothelial PECAM + cells were revealed by BrdU immunostaining, with BrdU being injected $1 \mathrm{~h}$ before animal perfusion. Orthogonal projection of a BrdU +/PECAM + cell is presented as viewed in the $x-z$ (bottom) and $y-z$ (right) planes. Note that proliferating endothelial cells (colocalization of PECAM/BrdU) were mostly found at the borders of the RMS. In contrast to early periods of development (P3), no angiogenesis was detected in adulthood (P60). G, Migrating endothelial "tip" cells with filopodia in the developing RMS visualized by PECAM immunostaining. $\boldsymbol{H}$, Micrographs showing nonfunctional BVs (arrows) at early stages of RMS development (P3). Colocalization between PECAM and dextran Texas Red indicates functional blood-carrying vessels. Note the presence of PECAM-positive but dextran Texas Red-negative nonfunctional blood vessels (arrows) in P3 RMS and their complete absence in the adult migratory stream. RMS is indicated by the dashed white line. I, Quantification of nonfunctional blood vessels in the RMS at different developmental periods. The values are presented as a percentage of length of PECAM+/dextran Texas Red - blood vessels from the total length of blood vessels. addition, no difference in astrocyte density derived from different brain regions was observed (Fig. $2 C, D$; cortex, $2.3 \pm 0.2$; cerebellum, $2.6 \pm 0.3$; SVZ-RMS, $2.2 \pm 0.1 ; \times 10^{3} \mathrm{cells} / \mu \mathrm{m}^{2} ; n=3$ cultures). These experiments indicated that astrocytes derived from the P3 SVZ-RMS, but not from P3 cortex and cerebellum or adult SVZ, secrete molecular cues that regulate the formation and growth of new BVs. But what is the nature of the secreted factor(s) that leads to the formation and growth of new BVs in the developing RMS and is downregulated in adulthood?

\section{RMS astrocytes synthesize and secrete VEGF}

VEGF, which is one of the major angiogenic molecules that controls vasculature development (Tammela et al., 2005; Grunewald et al., 2006), can be expressed by astrocytes (Stone et al., 1995; Sinor et al., 1998; Lee et al., 1999). We thus tested the putative role of VEGF in the appearance of RMS BVs during early developmental stages. We first investigated whether the potentiating role of P3 SVZ-RMS astrocyte-derived conditioning medium on the formation and growth of BVs was attributable to the presence of VEGF. We collected the conditioning medium from astrocytes derived from different $\mathrm{P} 3$ brain regions and cultured for $7 \mathrm{~d}$ and analyzed them by Western blotting. We have also analyzed the presence of VEGF in the conditioning medium derived from the adult SVZ astrocytes. Since adult SVZ astrocytes required a longer period of culturing to reach the confluence, we also immunoblotted the conditioning medium derived from the adult astrocytes cultured for $14 \mathrm{~d}$ and as a control P3 SVZ astrocyte. VEGF was detected in the conditioning medium derived from P3 SVZ-RMS astrocytes. Three bands (17, 22, and $25 \mathrm{kDa}$ ) (Fig. 2G) corresponding to VEGF120, VEGF164, and VEGF188 splice variants of VEGF (also called VEGF-A), the major isoforms secreted by most cell types, were detected (Robinson and Stringer, 2001; Tammela et al., 2005). Very little or no VEGF was expressed by astrocyte cultures derived from the cortex, cerebellum, or adult SVZ (Fig. 2G). These results suggested that P3 SVZ-RMS astrocytes synthesize and secrete VEGF, which is primarily downregulated during adulthood, and that the potentiating effect of $\mathrm{P} 3$ SVZ-RMS conditioning medium on the formation and growth of new BVs may be caused by the action of this trophic factor.

To explore the cellular origin of VEGF expression in vivo, we immunostained and in situ hybridized VEGF in combination with cell-type-specific markers such as GFAP for astrocytes, Dcx and PSA-NCAM for neuroblasts, and PECAM for BVs during different developmental stages (P3, P7, P14, P21, and adult). We also used GFAP-GFP and GAD67-GFP transgenic animals, which allow for the easy identification of astrocytes and neuroblasts, respectively. Our in situ hybridization (Fig. 3A) and immunostaining (Fig. 3D) showed that in the P3 SVZ-RMS, VEGF is expressed at the outer border of the RMS. Fluorescent in situ hybridization (Fig. $3 C$ ) and immunostaining (Fig. 3D,E) for VEGF in GFAP-GFP mice revealed that astrocytes in the developing RMS are the cellular source for this trophic factor. Interestingly, VEGF expression was developmentally regulated and massively decreased already at P14 (Fig. 3D). We were unable to detect any colocalization between VEGF and neuroblasts (identified by Dcx or PSA-NCAM immunolabeling) or in GAD67GFP mice. VEGF was also expressed in astrocyte cultures derived from the SVZ-RMS region (Fig. $3 F$ ). In contrast, fluorescent in situ hybridization and immunostaining for VEGF in the cortex and cerebellum revealed that astrocytes do not express this trophic factor, which appears to be expressed solely by neurons (Fig. $3 B, G$ ). In addition, immunostaining for VEGF in embryonic day 17 cortex also revealed a neuronal pattern of expression 

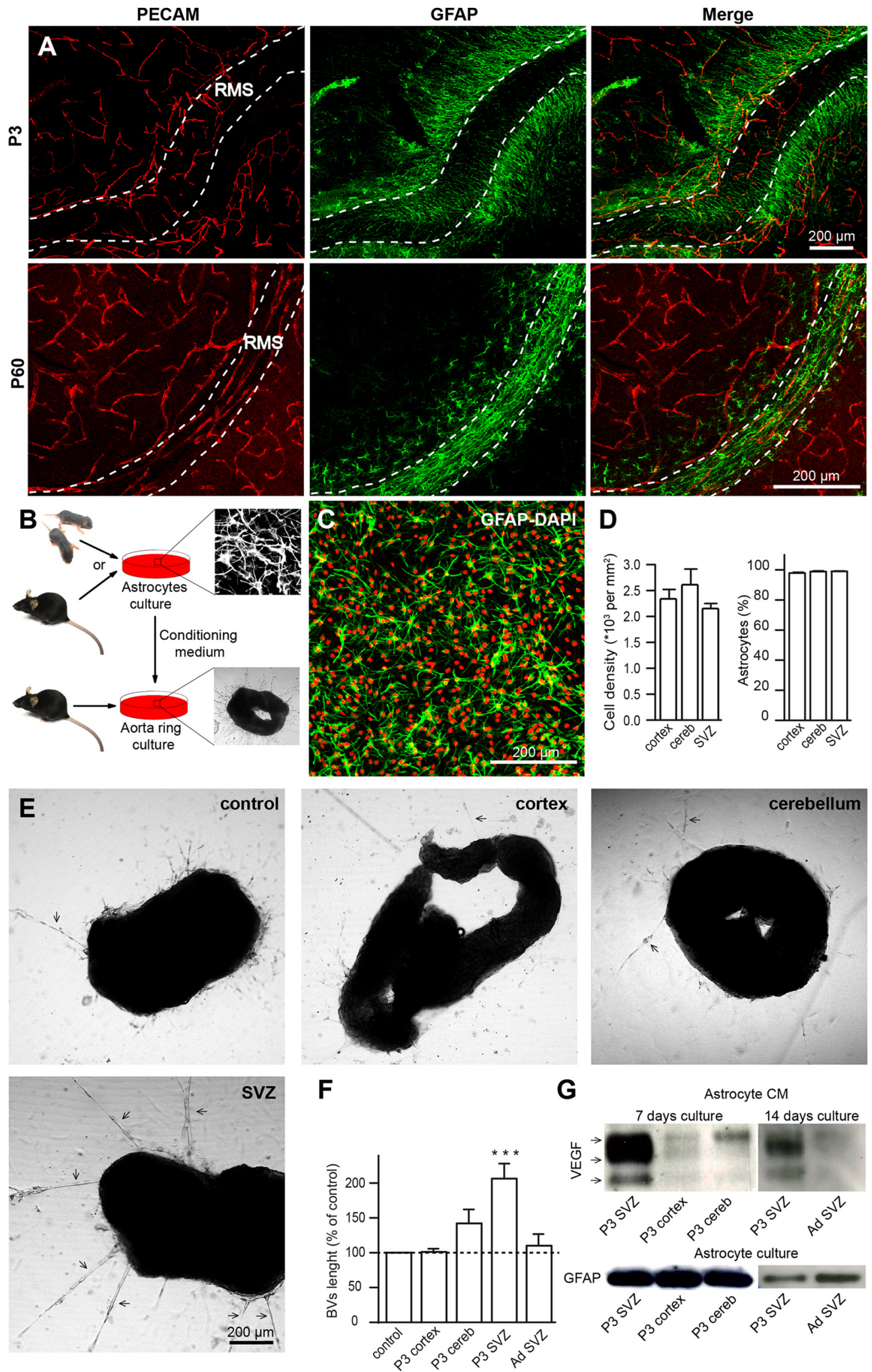

Figure 2. RMS astrocytes regulate the formation and growth of BVs A, Micrographs depicting PECAM + BVs (red) and GFAP + astrocytes (green) at the beginning of postnatal development (P3, top) and in adulthood (P60, bottom). Note that at the early developmental periods, astrocytes are positioned at the outer border of the RMS, whereas in adults they (Figure legend continues.) 
(data not shown). In agreement with our data, it has been previously demonstrated that VEGF in the cortex and cerebellum is expressed by cortical neurons and Purkinje cells, respectively (Ogunshola et al., 2002; Ruiz de Almodovar et al., 2010). This differential expression pattern of VEGF may explain the different effects of conditioning medium derived from cultures of astrocytes from P3 SVZ, cortex, cerebellum, and adult SVZ on BV development and growth (Fig. 2E,F).

We next decided to quantitatively evaluate VEGF expression in the RMS and explore how the expression pattern of this trophic factor changes during postnatal development. We dissected out the RMS under a fluorescent microscope from the acute slices prepared from GAD67-GFP or GFAP-GFP mice (which allow the easy identification of the RMS) and performed Western blot analyses. The major band of $17 \mathrm{kDa}$ corresponding to the VEGF120 splice variant of VEGF was detected in vivo, in the developing RMS. In line with our immunostaining results (Fig. $3 D$ ), our immunoblotting data showed that the expression of VEGF is developmentally regulated (Fig. $3 H$ ). This trophic factor was highly expressed during early developmental stages (P3-P7), whereas its expression was lower in adulthood (Fig. $3 \mathrm{H}, \mathrm{I}$; P7, $118.8 \pm 22.0 ; \mathrm{P} 14,8.8 \pm 0.5$; adult, $1.3 \pm 0.9$; the data were normalized to the GFAP level, and P3 was taken as $100 \% ; n=5$ animals per group). These experiments allowed us to establish the cellular pattern and developmental profile of VEGF expression.

\section{Astrocyte-derived VEGF is essential for BV development in the postnatal RMS}

To directly assess the role of VEGF in the formation and growth of BVs, we performed gain-of-function and loss-of-function experiments. For the gain-of-function experiments, we added different concentrations of exogenous $\operatorname{VEGF}(2,10,25$, and 100 $\mathrm{ng} / \mathrm{ml}$ ) to aorta ring cultures. Exogenous VEGF promoted the formation and growth of BVs in these cultures, with $10-25 \mathrm{ng} / \mathrm{ml}$ producing the most marked angiogenic effect (Fig. $4 A, B$; control: $100 \%, n=17$ cultures; $2 \mathrm{ng} / \mathrm{ml}: 167.65 \pm 21.4 \%, n=5$ cultures; $10 \mathrm{ng} / \mathrm{ml}: 276.5 \pm 59.8 \%, n=8$ cultures; $25 \mathrm{ng} / \mathrm{ml}: 288.23 \pm$ $32.7 \%, n=5$ cultures; $100 \mathrm{ng} / \mathrm{ml}: 165.4 \pm 44.7 \%, n=7$ cultures). To demonstrate that SVZ-RMS astrocyte-derived VEGF is involved in the formation and growth of BVs, we next performed

\footnotetext{
$\leftarrow$

(Figure legend continued.) are located inside of the migratory stream. RMS is indicated by the dashed white line. $\boldsymbol{B}$, In vitro method for studying the role of astrocytes on BV formation and growth in cultures. Astrocytes were prepared from the different brain regions of $P 3$ pups or from adult SVZ, and the ring of aorta was taken from adult mice. The conditioning medium (CM) derived from astrocytes was added to a 3D cultured aorta's rings every second day. C, Confocal image of cultured astrocytes. Astrocytes were stained with GFAP antibodies (green), and the cell nuclei were depicted by DAPI staining (red). Note that almost every DAPI-positive cell is colocalizing with GFAP, indicating high purity of astrocyte cultures. D, Quantification of cell density and purity of astrocyte cultures prepared from cortex, cerebellum, and SVZ-RMS. The first graph shows that there is no difference in the cell number of cultured astrocytes derived from different brain regions. The second graph shows the high purity of astrocyte cultures. $\boldsymbol{E}$, Representative images showing BV growth from the aortic ring in the absence (control) and presence of conditioning medium derived from astrocytes of different regions. Arrows indicate newly formed BVs. $\boldsymbol{F}$, Formation and growth of new blood vessels from the aortic ring after addition of conditioning medium from cortical, cerebellar, and SVZ astrocyte cultures. Note that only conditioning medium from P3 SVZ astrocytes potentiates formation and growth of new blood vessels. $\mathbf{G}$, Western blot analysis of astrocyte culture-derived conditioning medium (CM) showing that in contrast to P3 cortex, P3 cerebellum, and adult SVZ-RMS, P3 SVZ-RMS-derived astrocytes secrete VEGF. Since adult astrocytes require longer culturing to reach the confluence, we have also immunoblotted conditioning medium of $14 \mathrm{~d}$ cultured adult SVZ astrocytes. Astrocyte cultures from which conditioning medium was derived were also immunoblotted for GFAP to ascertain an equal amount of astrocytes used for experiment.
}

loss-of-function experiments with siRNA directed against VEGF as well as with control siRNA (Fig. $4 C, D$ ). The transfection of SVZ-RMS astrocytes with siRNA directed against VEGF reduced the expression of this trophic factor by $58.9 \pm 5.57 \%$ compared with the control siRNA-transfected cultures (Fig. $4 D$; the results were normalized to the amount of GFAP mRNA; $n=3$ cultures per condition). Conditioning medium derived from SVZ-RMS astrocytes transfected with siRNA directed against VEGF strongly reduced the formation and growth of BVs in aortic ring cultures compared with a nontransfected control or conditioning medium from cultures transfected with control siRNA (Fig. 4C,D; nontransfected, 100\%; control siRNA, $100.8 \pm 3.6 \%$; VEGF siRNA, $55.0 \pm 4.3 \% ; n=3$ cultures per condition). These in vitro experiments revealed that the VEGF secreted by RMS astrocytes plays a role in the angiogenesis observed during early postnatal development.

We next attempted to downregulate VEGF expression in vivo, specifically in astrocytes of the developing RMS, and to analyze $\mathrm{BV}$ formation. We constructed a lentivirus containing VEGF miRNA under the control of the GFAP promoter and injected it into two sites of the P3-P4 RMS (Fig. 5A). The lentiviral vector also contained GFP to make it easy to identify infected cells. To validate our virus, we first immunolabeled the cells with GFAP and Dcx, markers for astrocytes and neuroblasts, respectively (Fig. 5B-D). As shown in Figure 5, $B-D$, all the infected cells were colabeled with GFAP, but not Dcx, confirming that the lentivirus with the GFAP promoter specifically targeted astrocytes. Since in the RMS, GFAP immunostaining mostly reveals a dense network of astrocytic processes and does not fully stain the cell bodies, we also used GFAP-GFP and GAD67-GFP mice where astrocytes and neuroblasts, respectively, are homogenously filled with GFP. We injected into the RMS of these animals lentivirus expressing ZsGreen protein under the control of GFAP promoter. We then performed immunostaining using primary antibodies against GFP and ZsGreen and revealed the labels using a secondary antibody coupled to Alexa546 and Alexa633, respectively. These experiments (Fig. $5 E, F$ ) clearly demonstrate that ZsGreen-infected cells in the developing RMS are astrocytes, and not neuroblasts. In our control experiments, we established that anti-GFP antibody does not recognize ZsGreen and that anti-ZsGreen antibody does not recognize GFP (data not shown). To assess the level of VEGF downregulation in vivo, we next injected VEGF miRNA and control lentiviruses into the P3-P4 RMS and performed Western blot analyses for this trophic factor on GFP+ tissue samples dissected from the P14 migratory stream. Two different VEGF miRNA lentiviruses (VEGF miRNA 10 and VEGF miRNA 11) under the GFAP promoter were tested and induced, respectively, $36.8 \pm 7.0$ and $53.7 \pm 5.0 \%$ downregulation in the VEGF level compared with the control lentivirus (Fig. $6 \mathrm{~A}$, inset; $n=3-4$ animals per condition; the results were normalized to the amount of GFAP to ensure that the same number of astrocytes was used for the analyses).

Having validated the virus, we next investigated the structure of BVs in infected RMS where VEGF expression by astrocytes was downregulated. Intriguingly, in brains where RMS astrocytes were infected with VEGF miRNAs at P3-P4, we observed a marked alteration of the vasculature scaffold at P14-P15. The BVs were shorter and more branched, and the long parallel BVs that are normally present at the border of the RMS had vanished (Fig. 6A). We thus quantified the vasculature network in P14P15 brains infected either with VEGF miRNA or control viruses by analyzing the density of parallel and total BVs, as well as BV branching points. Our results revealed that VEGF downregula- 


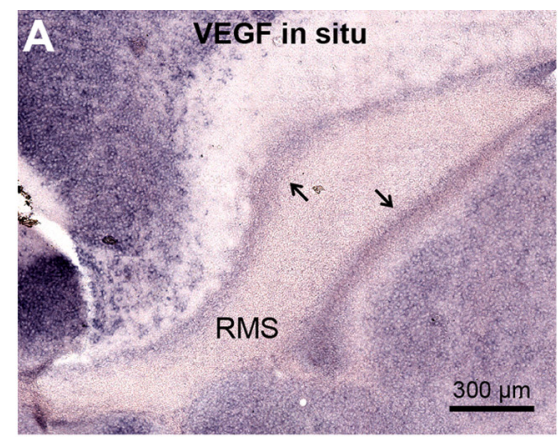

VEGF FISH/GFAP-GFP
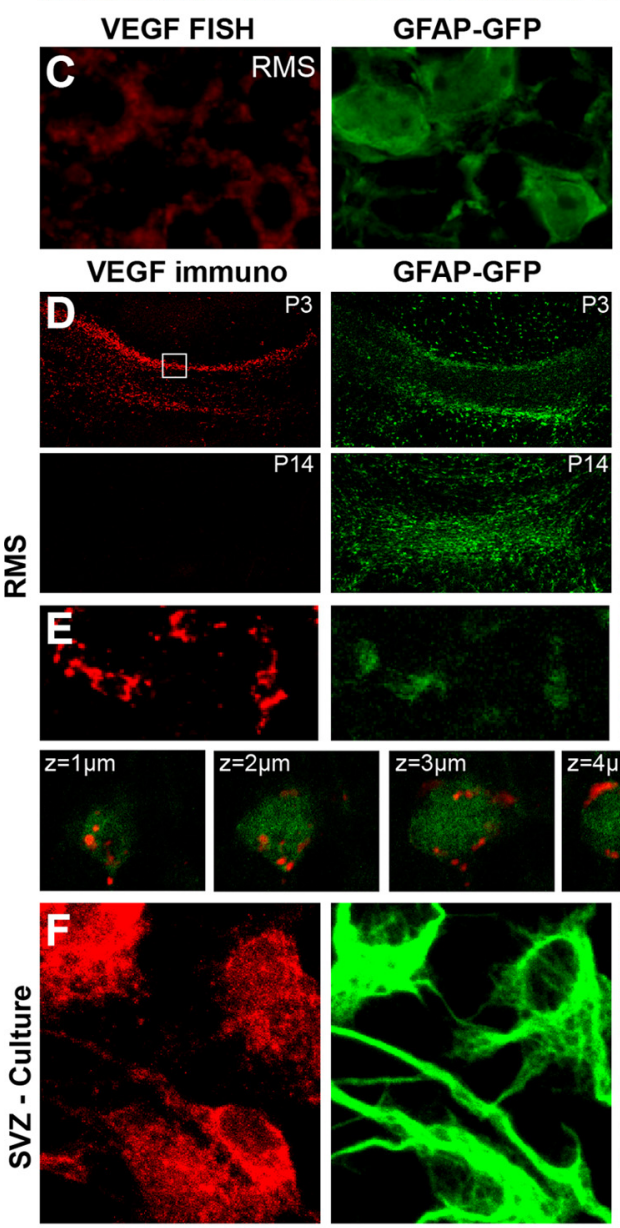

GFAP-GFP
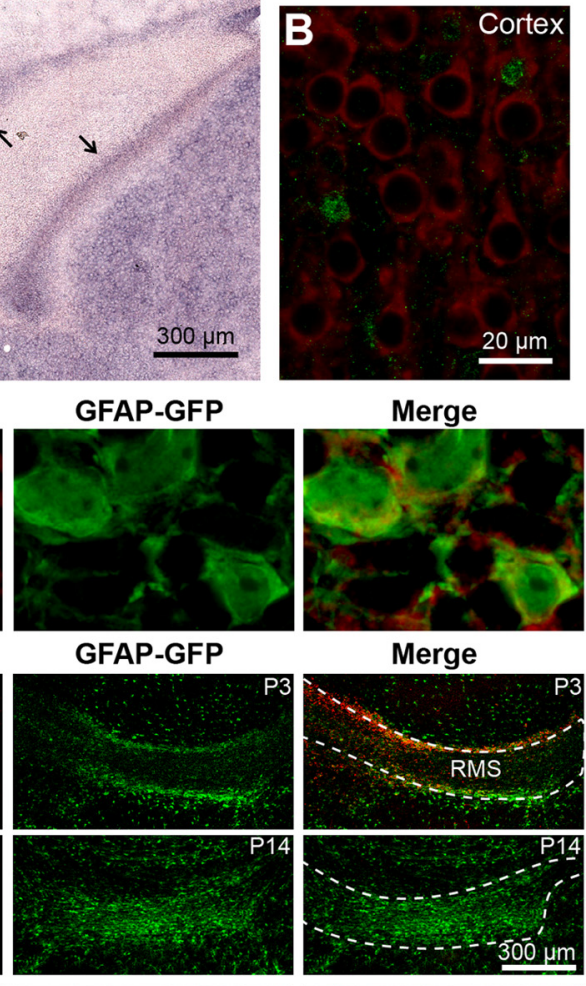

Merge
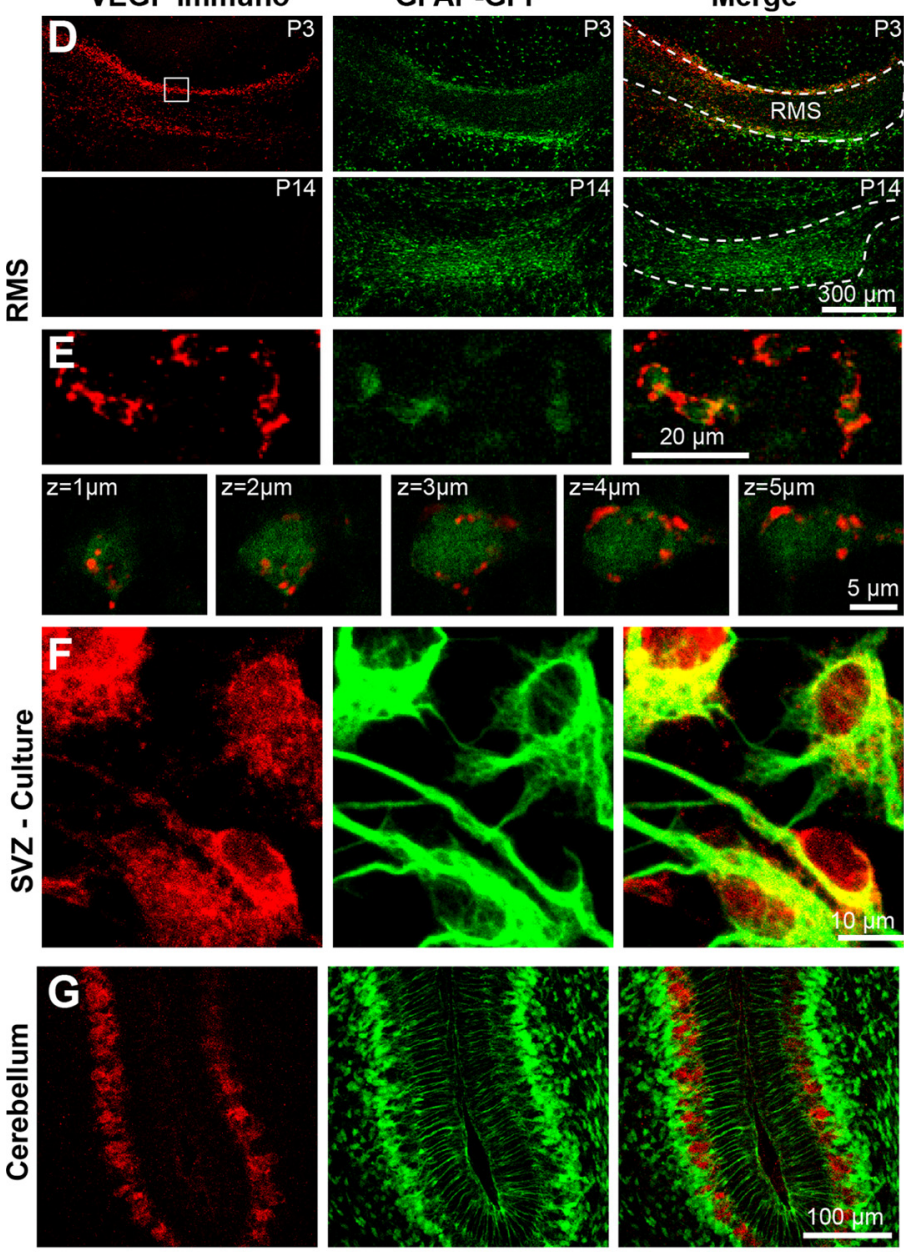

H
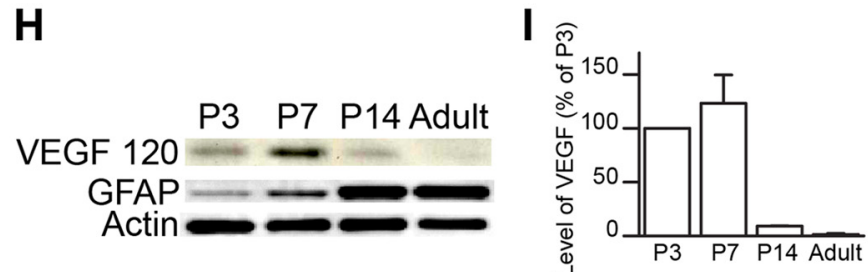

Figure 3. RMS astrocytes express VEGF in vivo and in vitro. $A$, VEGF mRNA expression pattern in P3 RMS. Note the high density of VEGF-expressing cells at the RMS borders (arrows) and absence of signal in the center of the migratory stream. $B, C$, Fluorescent in situ hybridization for VEGF (red) in the cortex $(\boldsymbol{B})$ and RMS $(\boldsymbol{C})$ of P3 GFAP-GFP mice (astrocytes in green). Note the VEGF tion in RMS astrocytes at P3-P4 with both VEGF miRNA constructs significantly decreases the overall BV density at the outer border of the RMS at P14-P15 compared with control miRNA-infected brains (Fig. $6 B$; control miRNA RMS, $0.75 \pm 0.025$; VEGF miRNA 10 RMS, $0.80 \pm 0.03$; VEGF miRNA 11 RMS, $0.7 \pm 0.07$; control miRNA RMS border, $1.30 \pm 0.05$; VEGF miRNA 10 RMS border, $1.0 \pm 0.03$; VEGF miRNA 11 RMS border, $0.85 \pm$ $0.03 \times 10^{3}$ per cubed micrometer; $n=3$ animals per group; $p<0.05$ between control miRNA RMS border and VEGF miRNA 10 and 11 RMS border with Student's $t$ test). The density of parallel BVs was also reduced at the outer border of RMS with the injection of lentiviruses having VEGF miRNAs under the GFAP promoter (Fig. 6C; control miRNA RMS, $0.45 \pm 0.02$; VEGF miRNA 10 RMS, $0.37 \pm$ 0.02; VEGF miRNA 11 RMS, $0.38 \pm 0.05$; control miRNA border, $0.95 \pm 0.05$; VEGF miRNA 10 RMS border, $0.51 \pm$ 0.04; VEGF miRNA 11 RMS border, $0.37 \pm 0.09 \times 10^{3}$ per cubed micrometer; $n=3$ animals per group; $p<0.05$ with Student's $t$ test). Interestingly, VEGF miRNAs affected the total and parallel BV density only on the RMS borders. This is likely attributable to the fact that at P3$\mathrm{P} 7$, the angiogenesis and the major BV network rearrangements are happening at the RMS borders and the RMS center contains three times less parallel BVs (Fig. 1). Thus, VEGF downregulation at this critical period of angiogenesis in the RMS is likely to affect BVs at the border. At later developmental stages when astrocytes appear in the RMS center, the VEGF expres-

mRNA expression by cortical neurons and not by astrocytes in $\mathrm{P} 3$ cortex. In contrast, in P3 RMS astrocytes express a high level of this trophic factor. $D-G$, Micrographs showing a VEGF protein expression pattern in vivo in the RMS ( $\boldsymbol{D}$, top, $\boldsymbol{E})$ and cerebellum $(\boldsymbol{G})$ in P3 GFAP-GFP mice and in vitro in SVZ-RMS astrocytes cultures $(\boldsymbol{F})$. In the RMS, VEGF is expressed by astrocytes (colocalization of VEGF and GFP). $D$, Note that VEGF is expressed mostly at the borders of P3 RMS (top) where the astrocytes are located and where the first parallel BVs appear. VEGF expression is drastically reduced at P14 when astrocytes appear in the center of the RMS (bottom). $\boldsymbol{E}$, Micrographs are higher-magnification images showing expression of VEGF by P3 RMS astrocytes. A different optical section of astrocyte-expressing VEGF is also shown (bottom). $\boldsymbol{G}$, In contrast to RMS, in the cerebellum VEGF is mostly expressed by neurons. $\boldsymbol{H}$, Western blot analysis for VEGF, GFAP, and actin in P3, P7, P14, and adult RMS samples. Note that VEGF expression is markedly decreased from P3 to P7 until adulthood. I, Quantification of VEGF expression level during RMS development. The data were normalized to the level of GFAP, and P3 values were taken as $100 \%$. 

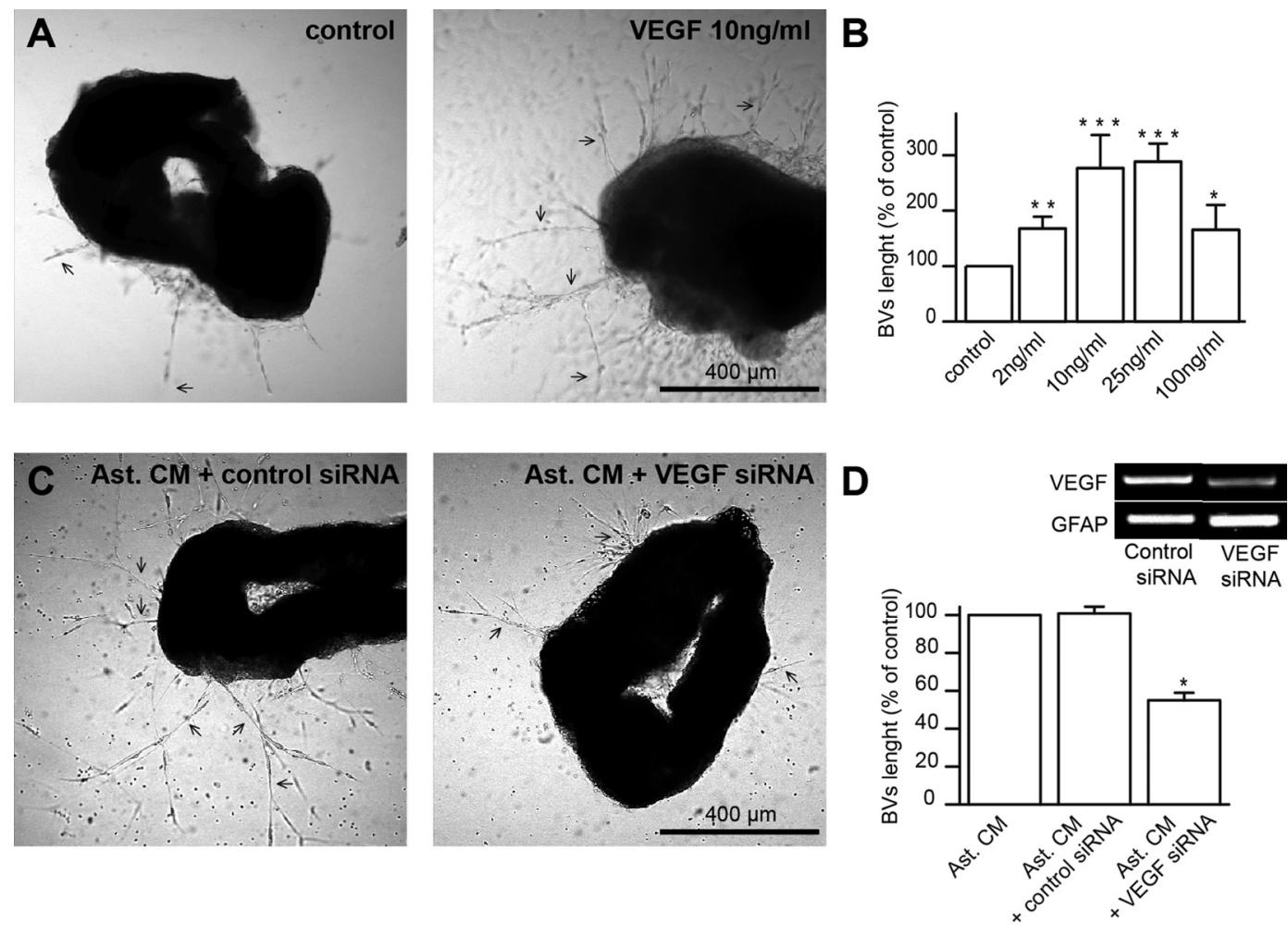

Figure 4. VEGF gain-and loss-of-function experiments affect the vasculature development. $A$, Representative images showing the newly formed BVs (arrows) in $3 D$ cultures of aortic rings under control conditions and after application of exogenous VEGF $(10 \mathrm{ng} / \mathrm{ml})$. B, Quantification of the length of newly formed BVs under the control condition and after application of VEGF at different concentrations. C, Representative images of aortic rings cultured with the conditioning medium derived from P3 SVZ astrocyte culture (Ast. CM) transfected with control and VEGF siRNAs. D, Quantification of BV length reveals almost 50\% reduction in cultures treated with conditioning medium derived from VEGF siRNA-transfected astrocytes compared with control conditions with conditioning medium derived from untransfected or control siRNA-transfected astrocytes. Inset, PCR analysis for VEGF and GFAP from astrocyte cultures transfected with control or VEGF siRNAs.

sion is decreased (Fig. 3D). The downregulation of VEGF also increased the value of BV branching points normalized to the density of BVs on RMS borders (Fig. 6D; control miRNA RMS, $9.13 \pm 0.7$; VEGF miRNA 10 RMS, $12.9 \pm 0.8$; VEGF miRNA 11 RMS, $9.43 \pm 0.15$; control miRNA border, $3.1 \pm 0.42$; VEGF miRNA 10 border, $4.9 \pm 0.4$; VEGF miRNA 11 RMS border, $5.15 \pm 0.83 \times 10^{3}$ per density of BVs; $n=3$ animals per group; $p<0.05$ between control miRNAs RMS border and VEGF miRNA RMS border). This increase is caused by the decrease of total BVs density, because the absolute number of BV branching points is not changed.

Thus, our in vitro and in vivo results from the gain- and loss-of function experiments clearly showed that VEGF is an essential factor for the development of the vasculature scaffold in the postnatal RMS. We previously showed that this vasculature scaffold is used by neuroblasts to migrate toward the OB in adulthood (Snapyan et al., 2009). However, the question remained as to how neuroblasts migrate at the beginning of postnatal development when the BV network in the RMS is not fully formed?

\section{Migration of neuroblasts during postnatal development}

Taking into account the important role of BVs in neuronal navigation in the adult brain (Snapyan et al., 2009; Whitman et al., 2009) and that at the early stages of RMS development the vasculature scaffold is still undergoing major reorganization, we decided to calculate the density and distance distribution of neuroblasts from BVs at different developmental ages and at distinct RMS subregions. The high density of neuroblasts migrating along the BVs has been previously shown for the adult RMS (Snapyan et al., 2009; Whitman et al., 2009). To calculate the density of neu- roblasts at $\mathrm{P} 5$ and $\mathrm{P} 14$, we injected retrovirus expressing GFP into the SVZ at P2 and P10, respectively, and quantified the number of $\mathrm{GFP}+$ neuroblasts at the border and center of the RMS (Fig. 7A). Interestingly, neuroblasts were localized at a higher density at the RMS border compared with the center of the migratory stream at both developmental ages (Fig. 7A; P5 RMS, $2.5 \pm 0.2 \times 10^{3}$ cells $/ \mathrm{mm}^{2}$; P5 RMS border, $3.2 \pm 0.3 \times 10^{3}$ cells $/ \mathrm{mm}^{2} ; n=1013$ and 494 cells from 3 animals, respectively; $p<0.05$ with paired $t$ test; P14 RMS, $2.4 \pm 0.1 \times 10^{3}$ cells $/ \mathrm{mm}^{2}$; P14 RMS border, $2.7 \pm 0.2 \times 10^{3}$ cells $/ \mathrm{mm}^{2} ; n=1254$ and 544 cells from 3 animals, respectively; $p=0.05$ with paired $t$ test). We then analyzed the distance of retrovirally labeled neuroblasts from dextran Texas Red filled BVs at different developmental ages (P5, P14, and P60) and at distinct subregions of the migratory stream (Fig. $7 A, B)$. Interestingly, over $80 \%$ of neuroblasts were positioned $<5 \mu \mathrm{m}$ away from BVs at the RMS borders at P5 and P14 and in the adult migratory stream (Fig. $7 B ; n=107,107$, and 228 cells for P5 RMS border, P14 RMS border, and adult RMS, respectively, of three animals). In contrast, in the center of the RMS at P5 and P14, only 10 and 30\% of neuroblasts, respectively, were located $<5 \mu \mathrm{m}$ away from BVs (Fig. $7 B ; n=228$ and 296 cells for P5 RMS and P14 RMS, respectively). These data indicate that neuroblasts are closely associated with vasculature scaffold at the RMS border, suggesting that they might preferentially migrate along BVs in this subregion of the migratory pathway. To provide direct evidence for vasculature-guided migration of neuronal precursors in the developing RMS, we performed real-time videoimaging experiments in the acute slices of P5 mouse forebrain. The fluorescent dextran Texas Red was injected into the blood stream shortly before preparation of acute slices to visualize the 

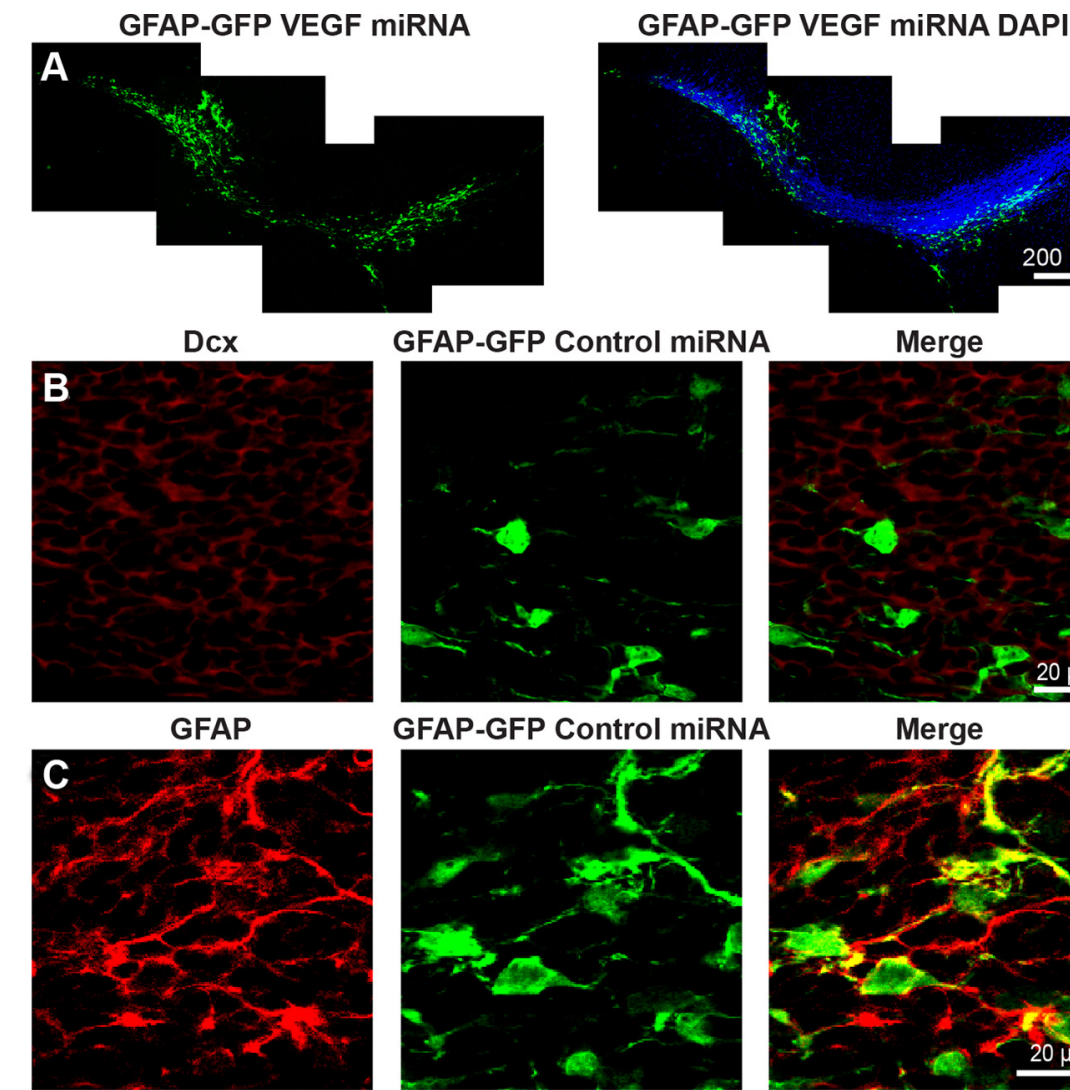

GFAP-GFP Control miRNA
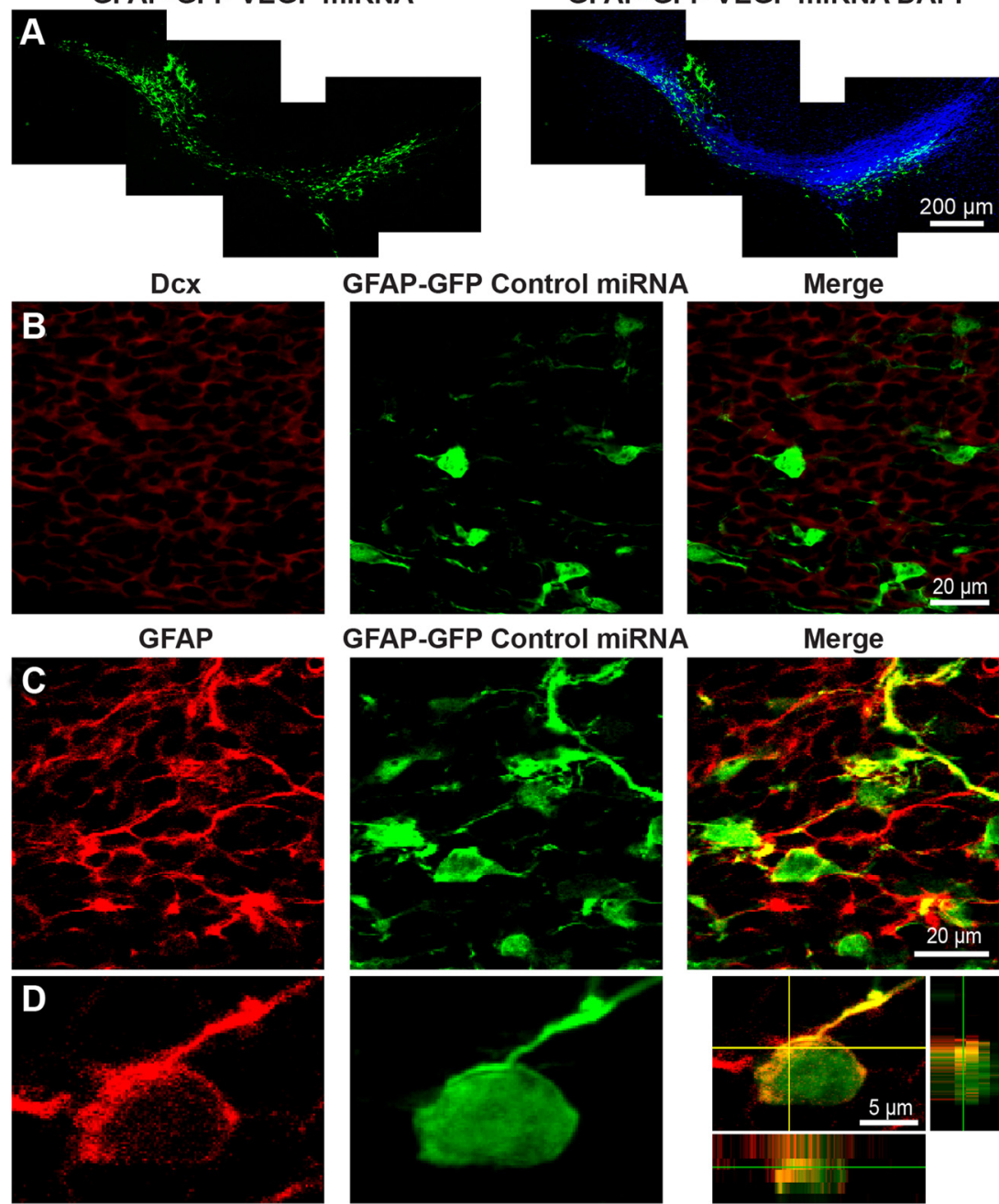

Injection of GFAP-ZsGreen virus into GFAP-GFP or GAD67-GFP RMS
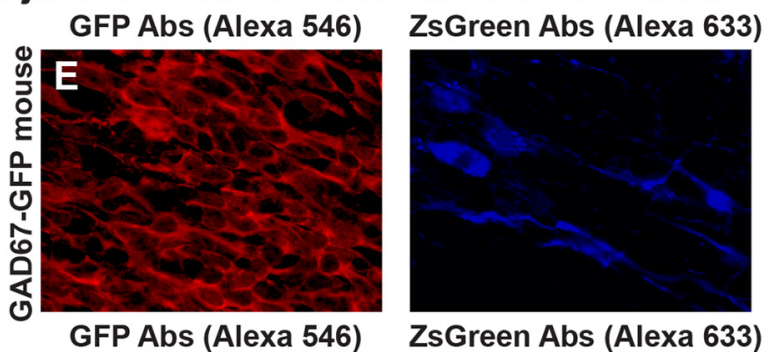

Merge
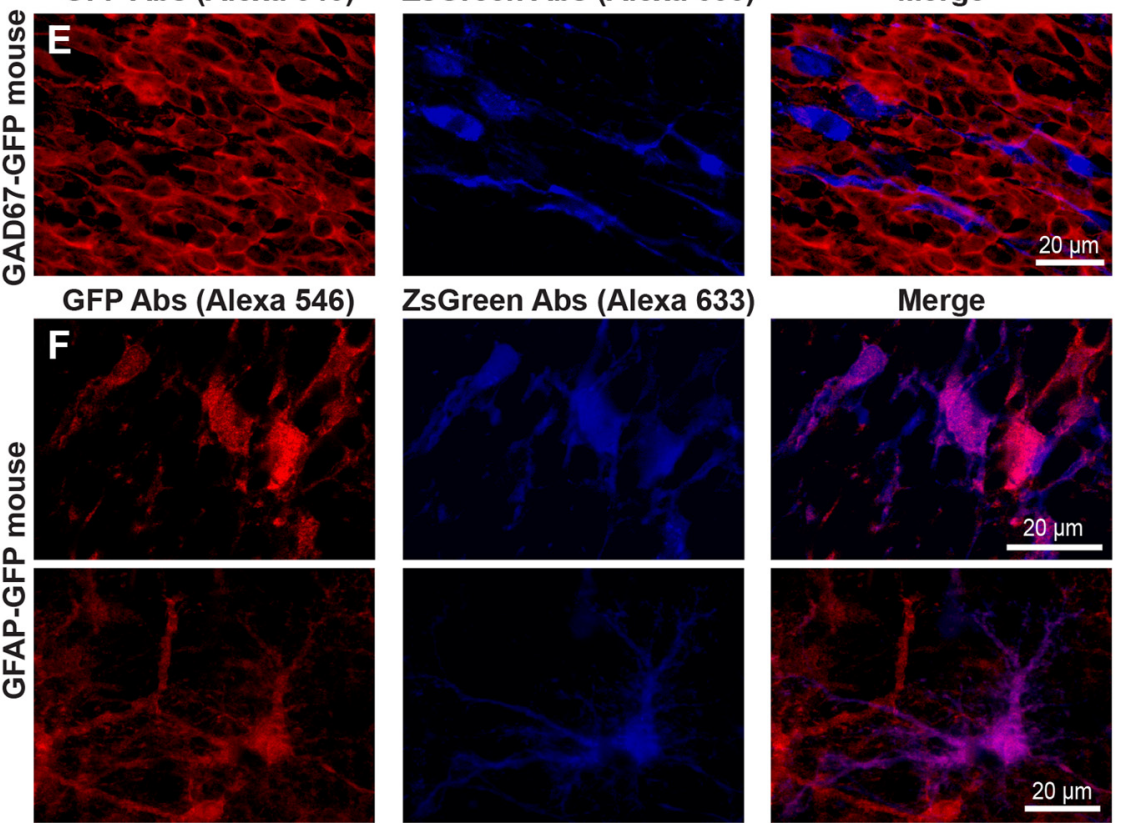

ZsGreen Abs (Alexa 633)
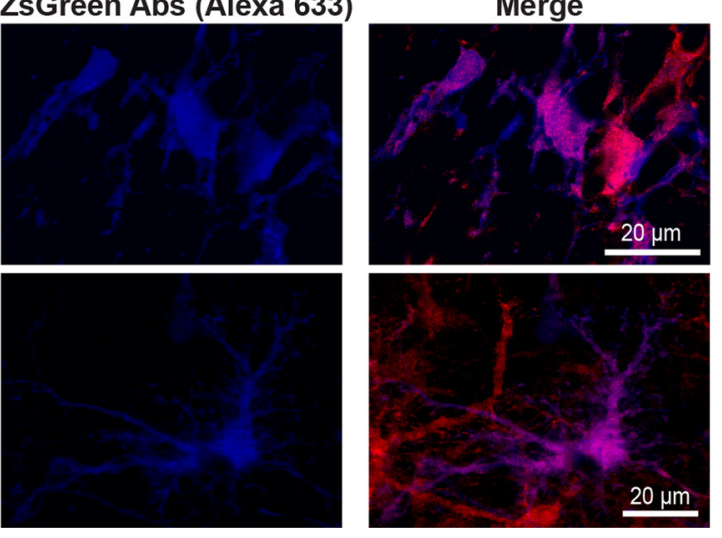

Figure 5. RMS-SVZ astrocytes can be specifically targeted in vivo. $A$, Confocal images demonstrating the high infection level after GFAP-GFP VEGF miRNA injection into two sites of P3 RMS. Infected cells are in green, and RMS was delimited by DAPI labeling (blue). $\boldsymbol{B}$, High-magnification images demonstrate absence of colocalization between Dcx (Figure legend continues.) 

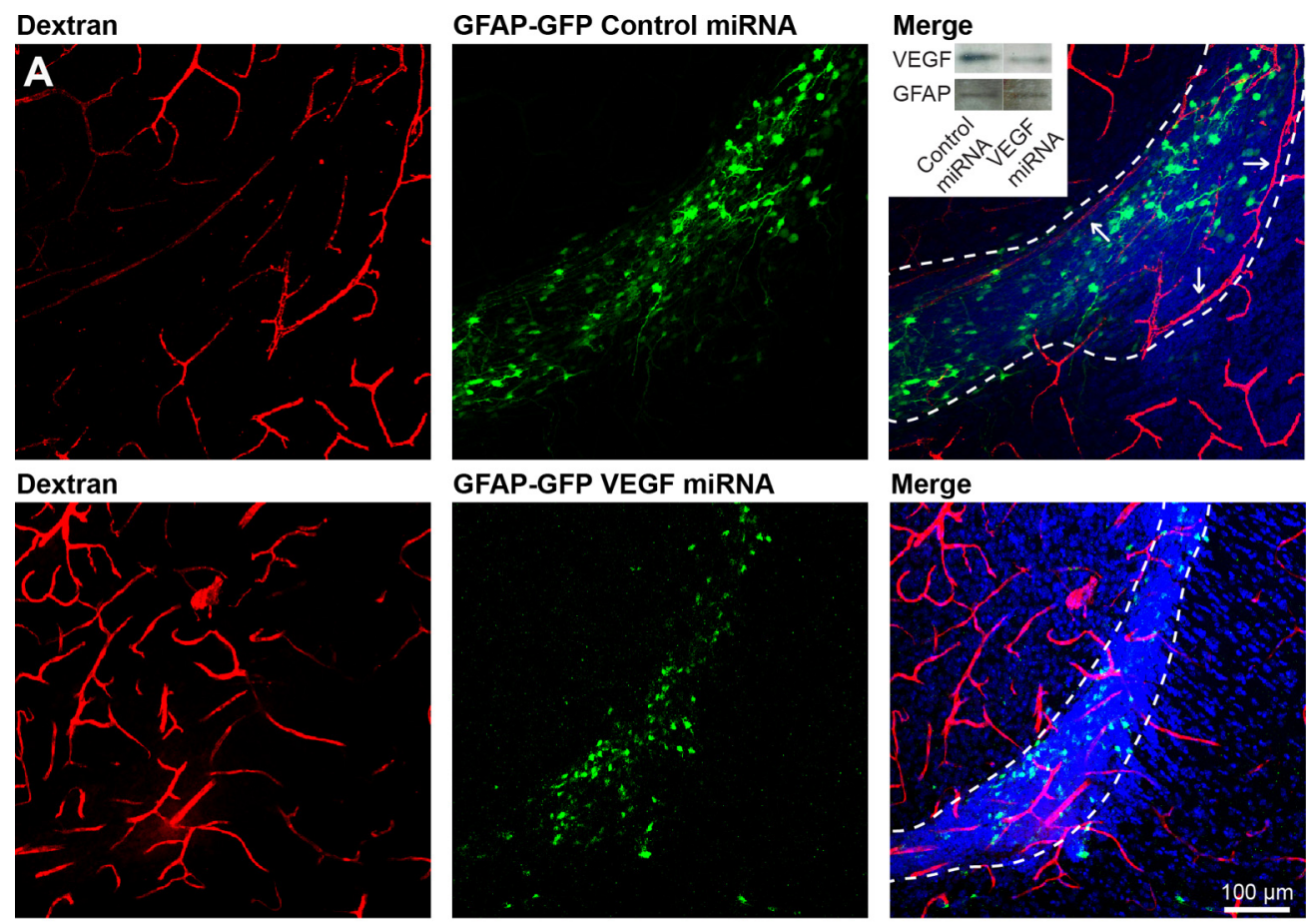

\section{Merge}
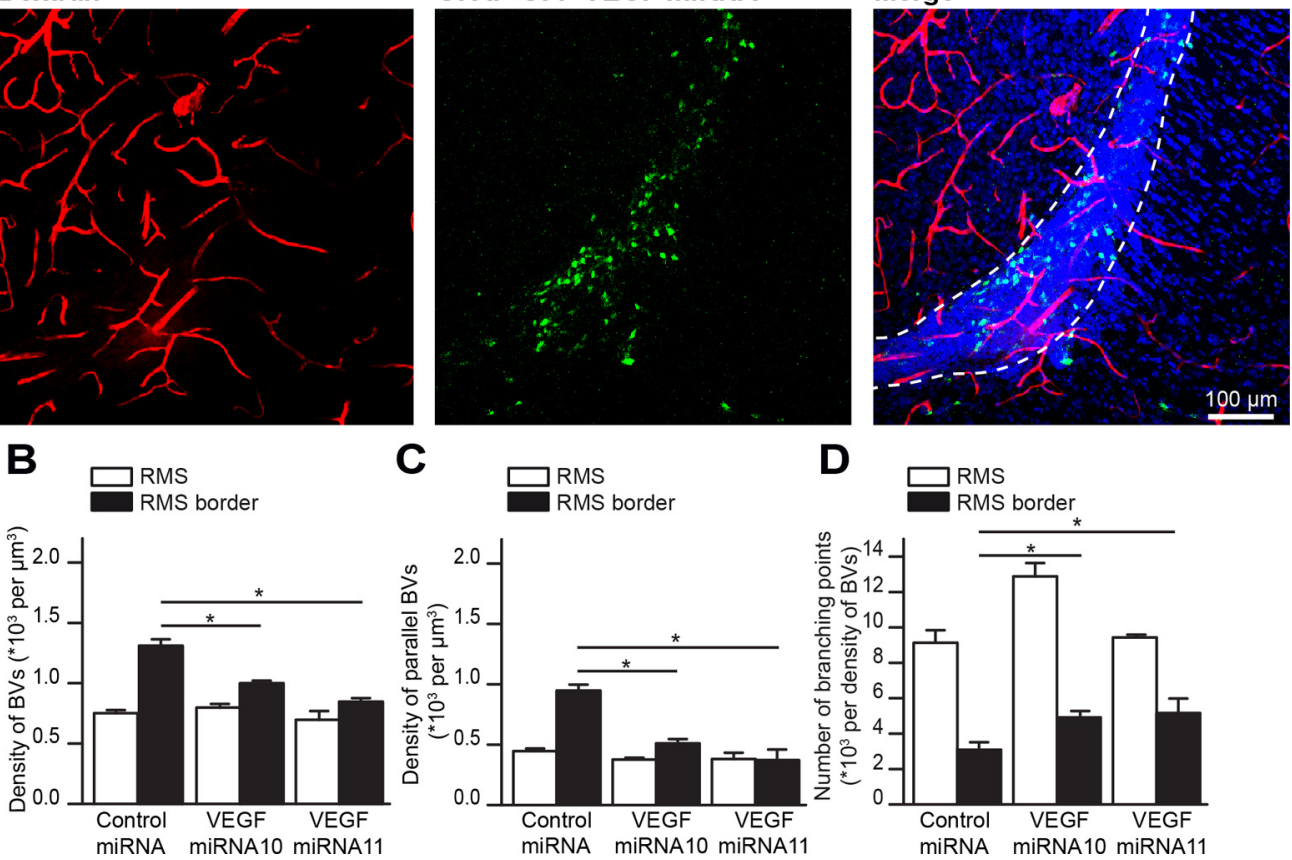

Figure 6. VEGF downregulation in RMS astrocytes alters the vasculature development in vivo. A, Profile of RMS vasculature at P15 after stereotaxic injection of control and VEGF miRNA viruses with GFAP promoter at P4. BVs were filled by dextran Texas Red (red). The viruses also drive GFP expression, allowing easy identification of infected cells (green). Nuclear DAPI staining (blue) is delimiting the RMS, which can be distinguished by the high density of cells. Note that with GFAP-GFP control miRNA, infected RMS contains more long and parallel BVs (arrows) with fewer sprouts compared with RMS infected with VEGF miRNA. Inset, Western blot analysis of VEGF downregulation in VEGF miRNA-injected RMS compared with the control miRNA-injected mice. Only the RMS regions containing GFP+-infected cells were dissected and used for immunoblotting. The data were normalized to GFAP level. $\boldsymbol{B}-\boldsymbol{D}$, Quantification of overall BV density ( $\boldsymbol{B})$, the density of parallel $\mathrm{BVs}(\boldsymbol{C})$, and BV branching points $(\boldsymbol{D})$ in the RMS and at its border at P15 after infection with control and VEGF miRNA viruses with GFAP promoter.

vasculature and neuroblasts were labeled by GFP-expressing retrovirus injection into the $\mathrm{SVZ}$ at $\mathrm{P} 2$. Our real-time videoimaging experiments reveal that retrovirally labeled neuroblasts remain close to the vasculature either with their soma or leading process during the entire period of recording (Fig. 7C). These data are reminiscent of the vasculature-guided migration of neuronal precursors observed in the adult RMS (Snapyan et al., 2009) and suggest that BVs serve as a scaffold for neuroblasts migration also in the developing migratory stream. However, since the vasculature scaffold during early developmental stages is still undergoing

$\leftarrow$

(Figure legend continued.) (red) and GFP-infected (green) cells. C, High-magnification images showing the colocalization of GFP-infected cells (green) with GFAP + astrocytes (red). D, Micrograph showing colocalization between GFP (green) and GFAP (red). Orthogonal projection of a GFP + /GFAP + cell is presented as viewed in the $x-z$ (bottom) and $y-z$ (right) planes. $\boldsymbol{E}, \boldsymbol{F}$, Injection of lentivirus expressing ZsGreen protein under the GFAP promoter into GFAP-GFP or GAD67-GFP RMS to ascertain the specific infection of astrocytes with the virus having GFAP promoter. The immunostaining with antibodies against GFP (red) and ZsGreen (blue) show that GFAP-ZsGreen virus infects only GFAP + astrocytes $(\boldsymbol{F})$ and not GAD67 + neuroblasts $(\boldsymbol{E})$. major reorganization, the density of BVs increases from $\mathrm{P} 3$ until adulthood, and neuroblasts located at the RMS center do not show vasculature association, we hypothesized that the migration of neuronal precursors in the developing RMS is less efficient than in the adult RMS. To test this hypothesis, we performed time-lapse imaging of cell migration in acute slices prepared from P4-P5, P13-P15, and P60 mice. Neuroblasts were labeled in the SVZ by stereotaxic injection of GFP-expressing retroviruses in P2, P10, and P57 mice (Fig. 7D). At all ages, neuroblast migration in the RMS was saltatory, with the migratory phases interrupted by periods of immobility (Fig. $7 D$ ). Time-lapse videoimaging revealed that the distance traveled by neuroblasts in the P5 RMS was significantly shorter than in the P14 and the adult RMS (Fig. $7 E$ ). Since neuroblasts use BVs as a scaffold for migration during development (Fig. 7C) and in adulthood (Snapyan et al., 2009) and since our morphological analysis revealed dramatic differences in the density of BVs (Fig. 1) and vasculature association of neuroblasts (Fig. $7 B$ ) at the outer border of and inside the RMS, we next analyzed cell migration in these two distinct compartments of the migratory stream. Interestingly, our analysis re- 


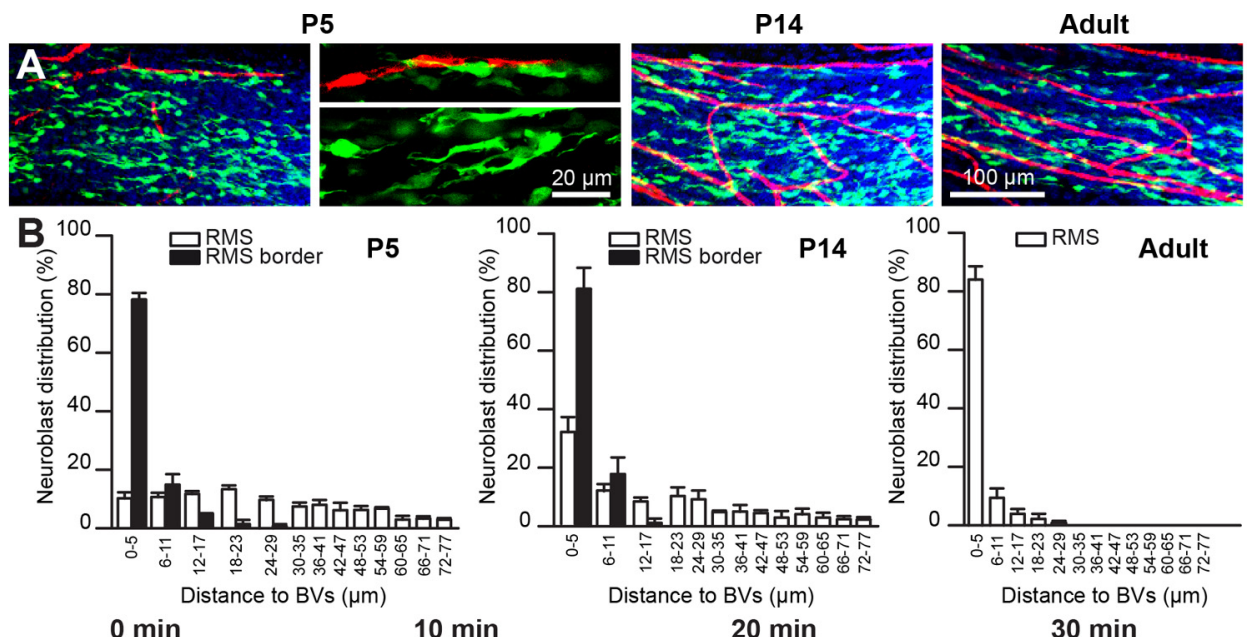

C
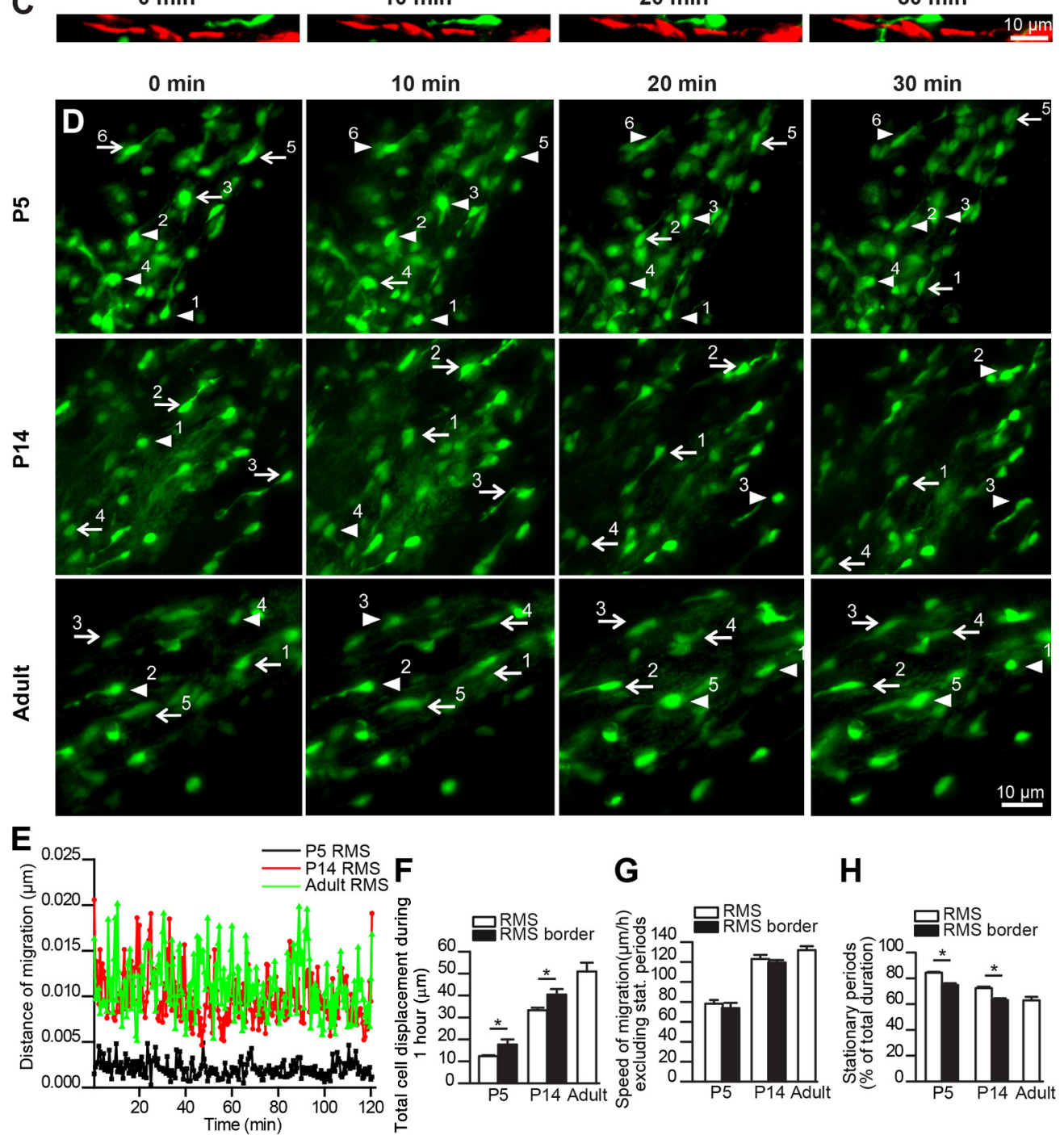

H

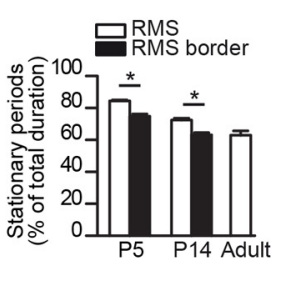

Figure 7. Neuroblast migration is less efficient at the beginning of postnatal development. $A$, Representative confocal images of retrovirally labeled neuroblasts (green) and BVs filled with dextran Texas Red (red) in P5, P14, and adult RMS showing the distribution of neuroblasts at the RMS center and border and their distance from BVs. Note that at P5 and P14, neuroblasts located at the RMS borders are closer to the BVs compared with those in the core of RMS. B, Summary graphs showing the distribution of the shortest distance of neuroblasts from BVs at different developmental stages. Note that at P5 and P14, over 80\% of neuroblasts in the RMS border are located $<5 \mu \mathrm{m}$ away from BVs. In contrast, in the RMS center, the distance between neuroblasts and BVs is much bigger and the distribution of the shortest distance of neuroblasts to BVs is more homogeneous. C, Real-time videoimaging of retrovirally labeled neuroblast (green) migrating on dextran Texas Red filled BV (red) in the acute slices of P5 mouse forebrain. D, Time-lapse videoimaging of migrating neuroblasts during RMS postnatal development. Slices were derived from P4/P5, P13-P15, and adultmouse forebrain. Migrating cells are indicated during their migratory (arrows) and stationary (arrowheads) phases. E, Migration distance of neuroblasts at different developmental stages. Note that at the early developmental periods, the displacement of neuronal precursors is significantly smaller. $\boldsymbol{F}$, Total distance propagated by neuronal precursors in the RMS at different developmental stages. Note that migration at the border of P5 and P14 RMS is more efficient than in the center of RMS. G, Rate of neuroblast migration in the RMS at different developmental stages. Although the rate of neuroblasts migration in P5 RMS is significantly smaller compared with P14 and adult RMS, no differences in the RMS subregions were observed. $\boldsymbol{H}$, Summary graph showing differences in the duration of stationary periods across the RMS subregions and developmental stages. 
vealed that the migration distance of neuroblasts is significantly higher at the border compared with those located inside of the P5 RMS (Fig. 7F; P5 RMS: $12.4 \pm 0.4 \mu \mathrm{m}, n=115$ cells from 3 animals; P5 border, $17.7 \pm 2.3 \mu \mathrm{m}, n=7$ cells from 2 animals; $p<0.05$ with paired $t$ test). The migration of neuroblasts at the border of the P14 migratory stream is also more efficient compared with neuroblasts migrating in the center of the RMS and resembles the migration in adulthood (Fig. 7F; P14 RMS: $33.3 \pm$ $1.1 \mu \mathrm{m}, n=112$ cells from 3 animals; P14 border, $40.5 \pm 2.5 \mu \mathrm{m}$, $n=19$ cells from 3 animals; $p<0.05$ with paired $t$ test; adult RMS: $51.0 \pm 4.0 \mu \mathrm{m} ; n=191$ cells from 3 animals). The difference in total displacement distance between the different developmental stages and distinct subregions of the migratory stream may be attributable either to changes in the rate of migration or the duration of the migratory phases. Since in our time-lapse videoimaging experiments we used relatively rapid acquisition (i.e., once per $30 \mathrm{~s}$ ), we could reliably quantify the duration of the migratory and stationary phases and assess the rate of migration exclusively during the migratory phases. Our analysis showed that although the rate of migration is lower at P5, there is no difference in the speed of neuroblast migration with respect to the RMS subregion (Fig. 7G; P5 RMS: $78.2 \pm 3.7 \mu \mathrm{m} / \mathrm{h}, n=115$ cells from 3 animals; P5 border: $73.8 \pm 5.2 \mu \mathrm{m} / \mathrm{h}, n=7$ cells from 2 animals; P14 RMS: $123.1 \pm 4.1 \mu \mathrm{m} / \mathrm{h}, n=112$ cells from 3 animals; P14 border: $119.5 \pm 2.5 \mu \mathrm{m} / \mathrm{h}, n=19$ cells from 3 animals; adult RMS: $131.9 \pm 4.0 \mu \mathrm{m} / \mathrm{h}, n=191$ cells from 3 animals). In contrast, neuroblasts had significantly longer stationary phases during early developmental stages. The duration of the stationary phases was also different at the border and center of the P5 and P14 RMS that contain a different density of parallel BVs (Fig. 7H; P5 RMS: $84.4 \pm 0.6 \%, n=115$ cells from 3 animals; P5 border: $74.8 \pm 1.4 \%, n=7$ cells from 2 animals; $p<0.05$ with paired $t$ test; P14 RMS: $72.4 \pm 1.2 \%, n=112$ cells from 3 animals; P14 border: $63.1 \pm 1.4 \%, n=19$ cells from 3 animals; $p<0.05$ with paired $t$ test; adult RMS: $56.84 \pm 0.94 \% ; n=191$ cells from 3 animals). These results showed that the vasculature scaffold plays an important role in neuronal navigation in both the developing and the adult RMS and that migration is more efficient in adulthood than in early developmental stages.

\section{In vivo downregulation of VEGF in RMS astrocytes alters neuroblast migration}

To directly address the role played by BVs in neuronal migration during RMS development, we used our in vivo VEGFdownregulation astrocyte model that alters the vasculature scaffold in the migratory stream (Fig. 6). We injected VEGF miRNA or control lentivirus into the P3-P4 RMS at two sites and analyzed neuronal migration at P15-P17, the period when major alterations in BV density were observed (Fig. 6). To visualize neuroblasts and track their migration, we also injected mCherryencoding lentivirus into the SVZ of the same animals at P10. Multichannel time-lapse videoimaging of mCherry+ neuroblasts and GFP + astrocytes in the RMS was performed on acute slices prepared from P15-P17 mice (Fig. 8A). In the RMS, where VEGF was downregulated and the BV network failed to develop properly, neuronal migration was strongly altered (Fig. 8A-E). Alterations in the vasculature scaffold by the downregulation of VEGF in astrocytes induced a twofold reduction in the overall displacement distance (Fig. $8 B, C$; control miRNA, $31.9 \pm 1.0$ $\mu \mathrm{m}$ vs VEGF miRNA, $16.7 \pm 1.3 \mu \mathrm{m} ; p<0.0001$ with Student's $t$ test; $n=97$ and 156 cells from 3 animals for control and VEGF miRNA, respectively). This reduction was caused by changes in the duration of the stationary phases (Fig. $8 \mathrm{E}$; control miRNA,
$71.4 \pm 0.6 \%$ vs VEGF miRNA, $85.1 \pm 1.1 \% ; p<0.0001$ with Student's $t$ test; $n=97$ and 156 cells from 3 animals for control and VEGF miRNA, respectively) but not in the speed of migration (Fig. $8 D$; control miRNA, $134.6 \pm 2.2 \mu \mathrm{m} / \mathrm{h}$ vs VEGF miRNA, $128.8 \pm 2.4 \mu \mathrm{m} / \mathrm{h} ; n=97$ and 156 cells from 3 animals for control and VEGF miRNA, respectively). These results indicated that the downregulation of VEGF in the astrocytes of the developing RMS strongly alters the migration of neuronal precursors. Since the same procedure affected BV development in the RMS (Fig. 6) and since the vasculature scaffold was used by neuroblasts for migration in the developing and the adult RMS (Fig. 7) (Snapyan et al., 2009; Whitman et al., 2009), we propose that the effect on neuroblast migration is caused by alterations in the vasculature scaffold. However, it is possible that VEGF acts directly on neuroblasts, and its downregulation in the developing RMS may thus decrease the migration of neuronal precursors. To rule out this possibility, we recorded the migration of neuroblast precursors in P15 RMS slices for $1 \mathrm{~h}$. We then added VEGF and recorded the migration of neuronal precursors for 1 additional hour. We reasoned that if the alteration in neuroblast migration after VEGF downregulation in astrocytes is caused by the direct effect of this trophic factor on the migrating neuronal precursors, then adding exogenous VEGF should potentiate neuroblast migration. However, the addition of VEGF did not induce a change in the total displacement distance (Fig. 8C; ACSF, $29.9 \pm 3.4 \mu \mathrm{m}$ vs VEGF, $31.5 \pm 1.8 \mu \mathrm{m} ; n=196$ and 171 cells from 3 animals for ACSF and VEGF, respectively), rate of migration (Fig. $8 \mathrm{D}$; ACSF, $136.6 \pm 1.2 \mu \mathrm{m} / \mathrm{h}$ vs VEGF, $137.9 \pm 2.0 ; n=196$ and 171 cells from 3 animals for ACSF and VEGF, respectively), or duration of the stationary phases (Fig. $8 \mathrm{E}$; ACSF, $77.4 \pm 1.6$ vs VEGF, $75.9 \pm$ 1.4; $n=196$ and 171 cells from 3 animals for ACSF and VEGF, respectively).

It is also possible that VEGF downregulation in astrocytes affects the glial scaffold that indirectly may alter neuronal migration. To rule out this possibility, we established an in vitro system where we can study the role of VEGF downregulation in astrocytes on neuronal migration without any confounding implication from the BVs. We thus cocultured either control or VEGF miRNA-infected astrocytes with RMS explants (Fig. $8 F$ ). We again did not detect any differences in the length of neuroblast chains migrated from the explants cocultured either with control or VEGF miRNA-infected astrocytes (control miRNA, $76.7 \pm 3.2$ $\mu \mathrm{m}$; VEGF miRNA, $73.2 \pm 2.76 \mu \mathrm{m} ; n=9$ explants per condition) (Fig. $8 F$ ). These data demonstrate that VEGF downregulation in astrocytes does not alter neuronal migration directly and that alterations in neuroblast migration observed in slices are attributable to the affected vasculature scaffold. If this is the case, then the association of neuroblasts with BVs should be decreased in VEGF miRNA-injected mice. To verify this hypothesis, we labeled neuroblasts by mCherry-expressing lentivirus injection into the SVZ at P12 and analyzed their distance from BVs filled with dextran Cascade Blue at P16-P17 in control and VEGF miRNA-injected animals (injection at P3). The quantification showed a significant decrease in the number of neuroblasts, which were located $<5 \mu \mathrm{m}$ away from BVs (Fig. $8 G ; n=403,458$, and 579 cells for naive, control miRNA, and VEGF miRNA, respectively; $n=3$ animals). Accordingly, the distance between neuroblasts and BVs was increased in VEGF miRNA-injected mice compared with control miRNA-injected or noninjected (naive) mice (Fig. 8G). Therefore, these data support the idea that alterations in neuroblast migration after astrocyte-specific downregulation of VEGF is caused by the affected vasculature scaffold. 

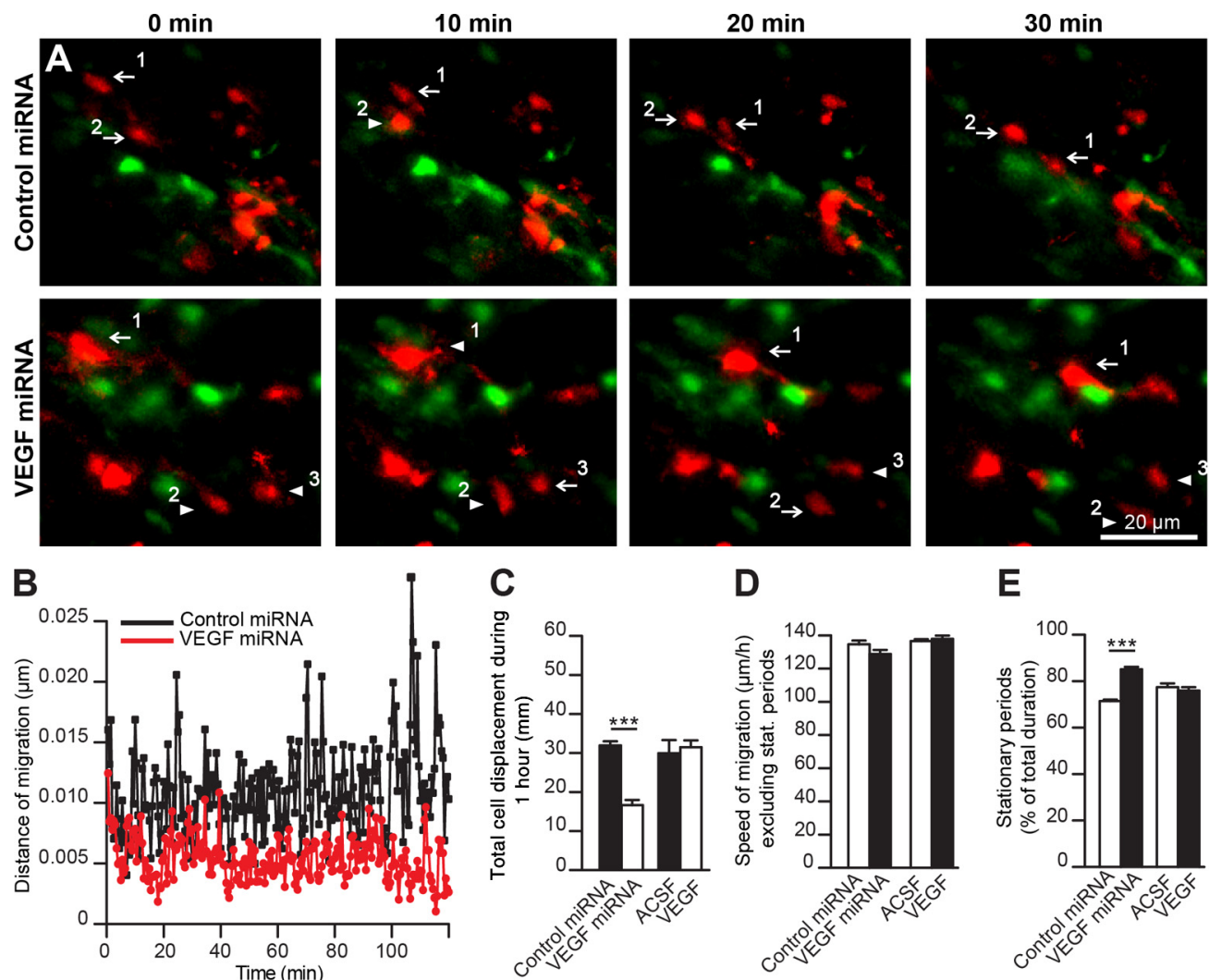

D

E

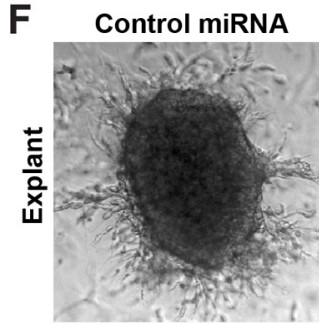

VEGF MIRNA
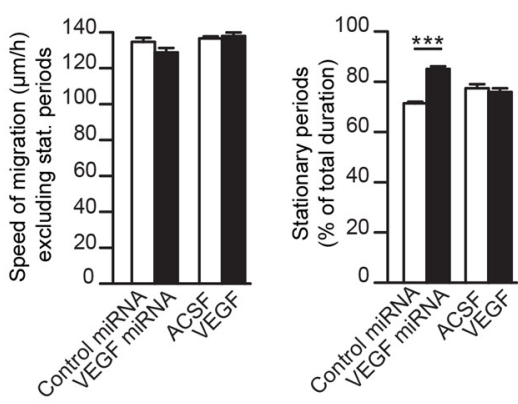

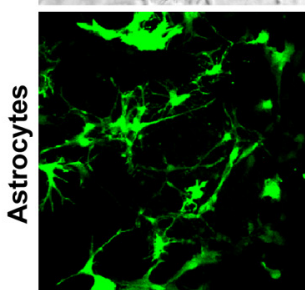

H

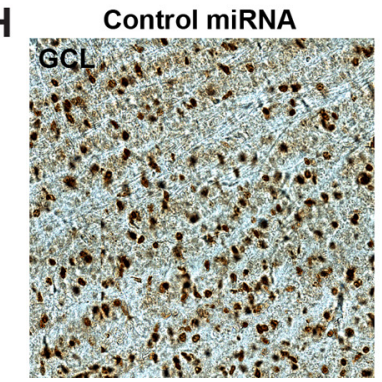

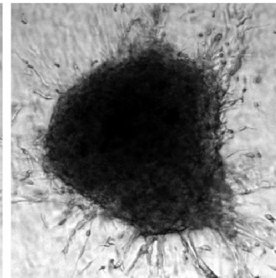

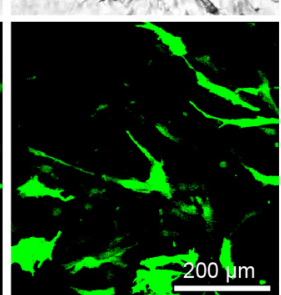

VEGF MIRNA

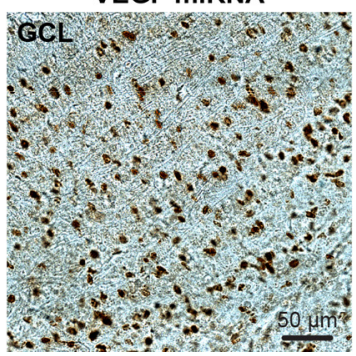

G
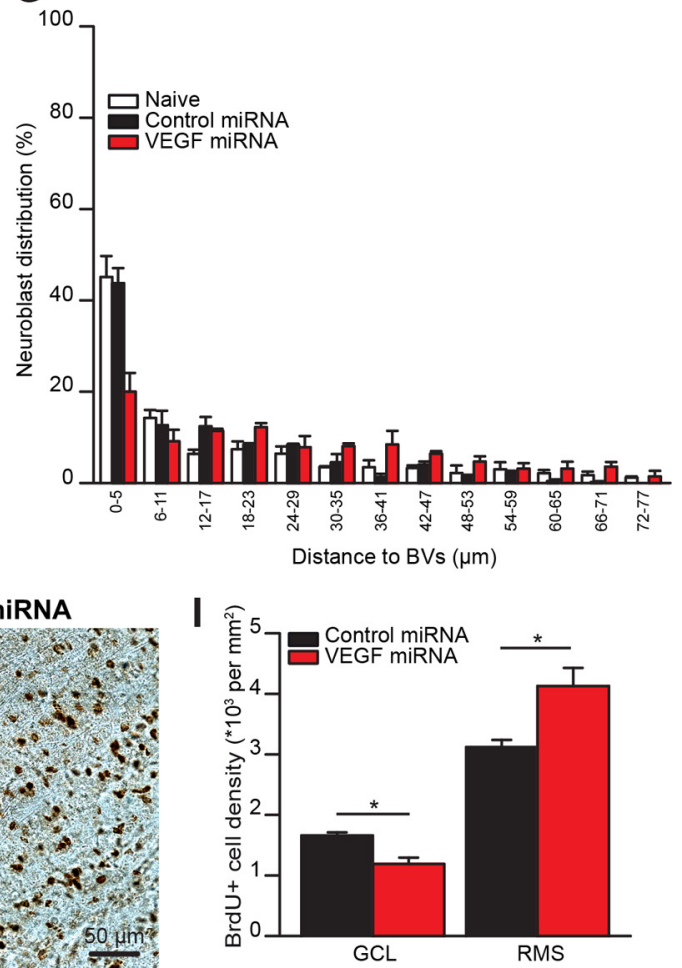

Figure 8. VEGF downregulation at the early postnatal developmental stages affects the migration of neuroblasts. $\boldsymbol{A}$, Time-lapse videoimaging of mCherry + migrating neuroblasts (red) at $\mathrm{P} 15$ after stereotaxic injection of control or VEGF miRNA lentiviruses with GFAP promoter (green) at P4 RMS. Migrating cells were labeled by stereotaxic injection of mCherry-expressing lentiviruses into P10 SVZ. Migrating cells are indicated during their migratory (arrows) and stationary (arrowheads) phases. B, Summary graph illustrating that the migration distance of neuroblasts is significantly decreased when the astrocytes were infected with VEGF miRNA. C, Total cell displacement of neuroblasts in P15 RMS after stereotaxic injection of control or miVEGF lentiviruses at P4. Note the reduction in the cell displacement after downregulation of VEGF in RMS astrocytes. In contrast, no changes in the migration of neuronal precursors were observed after acute VEGF application, suggesting that this trophic factor does not have any direct role on neuroblasts migration. $\boldsymbol{D}$, Unaltered rate of neuroblast migration in the RMS after VEGF downregulation in astrocytes at early developmental periods and after acute VEGF application. $\boldsymbol{E}$, Summary graph illustrating that the duration of the stationary periods is significantly higher in the RMS where VEGF was downregulated in the astrocytes. In contrast, acute VEGF application does not induce any effect on neuroblasts migration. $F$, Representative images of RMS explants (top) cocultured with astrocytes infected either with control or VEGF miRNA. The miRNAs viruses also express GFP, which allows the easy identification of infected cells (bottom). No difference in the length of neuroblast (Figure legend continues.) 
To provide further support for the migrational defects in mice with altered vasculature scaffold, we injected BrdU at P12 in control or VEGF miRNA-injected mice and quantified the density of BrdU+ cells in the GCL of the OB and RMS $7 \mathrm{~d}$ later (Fig. $8 H$ ). Notably, the density of BrdU+ cells was significantly decreased in the GCL (control miRNA, $1.66 \pm 0.05 \times 10^{3}$ per square millimeter; VEGF miRNA, $1.15 \pm 0.14 \times 10^{3}$ per square millimeter; $n=$ 3 animals; $p<0.05$ with Student's $t$ test), which was associated with the concomitant increase in the density of BrdU+ cells in the RMS, at the sites of VEGF miRNA injections (control miRNA, $3.12 \pm 0.18 \times 10^{3}$ per square millimeter; VEGF miRNA, $4.24 \pm 0.39 \times 10^{3}$ per square millimeter; $n=3$ animals; $p<0.05$ with Student's $t$ test) (Fig. $8 H, I)$. The altered distribution of BrdU+ cells along the SVZ-OB pathway strongly suggests the affected migration of neuronal precursors. In addition, to rule out the possibility that this increase in the density of BrdU + cells is attributable to affected proliferation, we quantified the density of Ki67+ cells in control and VEGF miRNA-injected mice. No difference in the density of Ki67+ cells was observed in the RMS of control and VEGF miRNA-injected mice (control miRNA, $7.96 \pm 0.290 .18-10^{3}$ per square millimeter; VEGF miRNA, $7.99 \pm 0.18 \times 10^{3}$ per square millimeter; $n=3$ animals). These results support the idea that the lower cell density in the $\mathrm{OB}$ as well as the accumulation of BrdU + cells in the RMS is caused by the alteration in vasculature scaffold, which is essential for neuronal migration.

Altogether, these data revealed that BVs play an essential role in neuroblast migration in the postnatal brain and highlight the fact that vasculature-associated migration occurs not only in the adult RMS but also during postnatal development.

\section{Discussion}

The goal of the present study was to gain a better understanding of the mechanisms of migratory stream development and neuroblast migration in the postnatal brain. We showed that astrocytes synthesize and secrete VEGF, which controls BV formation and growth. The vasculature scaffold is, in turn, used by neuroblasts for their migration toward the OB. We showed that, during postnatal development, the interplay between neuroblasts, astrocytes, and endothelial cells is important for the proper RMS development. We also revealed that this complex tripartite interplay occurs at the outer border of the migratory stream. We propose that the developing RMS is composed of two distinct subregions and that the cellular mechanisms regulating neuroblast migration during adulthood are laid down at the border of the developing migratory stream.

\section{Development of the postnatal migratory stream}

The RMS undergoes marked reorganization processes during early postnatal development. Glial, neuronal, and vascular com-

$\leftarrow$

(Figure legend continued.) chains arising from explants cocultured with control or VEGF miRNA was observed. G, Graphs showing the distribution of shortest distance of neuroblasts from BVs in control, VEGF miRNAs-injected animals, and in naive (noninjected) mice. miRNAs were injected at P3, and mCherry-expressing lentivirus was injected into the SVZ at P12. Note that the distance between neuroblasts and BVs is increasing when VEGF is downregulated in the RMS astrocytes. $\boldsymbol{H}$, Micrographs illustrating the reduced number of BrdU + cells in the GCL of the $O B$ after alteration in the vasculature scaffold by VEGF downregulation in the RMS astrocytes. I, Summary graphs illustrating a decrease in the density of BrdU + cells in GCL of OB and an accumulation of neuronal precursors in the RMS in VEGF miRNA-injected mice. Altered distribution of BrdU + cells along SVZ-OB pathway suggests affected migration of neuronal precursors. ponents of the RMS differentiate and/or develop postnatally. We showed that astrocytes are first located at the outer border of the RMS, the region that harbors a high density of BVs and where the first parallel BVs appear. It has been shown previously that RMS astrocytes are derived from radial glia (Alves et al., 2002) and locate at the RMS outer border during the first 2 postnatal weeks (Law et al., 1999; Peretto et al., 2005), that the vasculature undergoes reorganization during postnatal development (Colín-Castelán et al., 2011), and that proliferative cells are mostly located at the border of the migratory stream (Pencea and Luskin, 2003). Our results, together with those of previous reports, thus strongly suggest that the developing RMS is compartmentalized, with its central, more "static" region containing a high density of nonproliferative and barely migrating neuroblasts and its outer border displaying active cellular reorganization. The interplay between astrocytes, endothelial cells, and neuroblasts at the outer border of the RMS leads to the development of a migratory stream that strikingly resembles the cellular organization observed in the adult RMS. As in adulthood (Snapyan et al., 2009; Whitman et al., 2009), the outer border of the developing RMS contained neuroblasts migrating along the parallely organized BVs that are formed in astrocyte-containing areas. The presence of this vasculature scaffold raises an important question about the molecular mechanisms regulating the formation of long parallel BVs. We showed that, because of the specific location of VEGF-expressing astrocytes at the outer border of the developing RMS, this subregion of the migratory pathway contains a highly permissive angiogenic environment, interrupted by the nonangiogenic, VEGF-lacking center of the RMS. We propose that this molecular compartmentalization in the developing RMS leads to the formation and growth of BVs preferentially at the VEGF-containing outer border of the RMS. These newly forming BVs remain restricted to this narrow region of the RMS border, which eventually leads to the development of parallel blood vessels. These BVs are then used by neuroblasts for their migration toward the OB, and this cellular organization is preserved until adulthood.

However, this model raises another important question. That is, how this particular cellular organization with parallely organized BVs and astrocytes is "transferred" into the core of the RMS? This issue is especially important since our time-lapse videoimaging experiments in GFAP-GFP and Tie2-GFP mice to track astrocytes and endothelial cells, respectively, did not reveal any cellular translocation from the border to the core of the migratory stream (data not shown). Our results showed that, although the width of the migratory stream decreases throughout development, overall RMS volume, from the SVZ to the OB, remains stable. This might be attributable to overall brain growth during postnatal development, leading to the RMS elongation and thinning. It is thus conceivable that, because of these major structural modifications during overall brain growth, the center of the migratory stream collapses, leading to the maintenance of the outer border of the RMS until adulthood.

\section{The role of vasculature in the migration of newborn cells in the postnatal RMS}

Our results revealed that the parallel organization of the BV network in the adult RMS that serves as a scaffold for neuroblast migration (Snapyan et al., 2009; Whitman et al., 2009) does not exist throughout the RMS during the early stages of development. We showed that whereas neuroblast migration in the adult migratory stream is generally more efficient than in the developing RMS, the presence of parallel BVs also facilitates cell navigation during development. During the early stages of development, as 
well as in adulthood (Snapyan et al., 2009), the vasculature scaffold promotes the entry of neuroblasts into the migratory phase at the expense of the stationary phase. The durations of the stationary phases of the two distinct subregions in the developing RMS, which had different BV densities, were different at both P5 and P14. In the center of the RMS, neuroblasts had very short migratory phases intercepted by long stationary phases. In contrast, the durations of the migratory phases at the border of the developing RMS were longer. Interestingly, however, although the density of parallel BVs in P3 and P14 RMS borders were quite similar, we observed marked differences in the total cell displacement and the duration of migratory phases. This could be explained by higher overall density and lower branching of BVs in P14 compared with P3 RMS. We observed no differences in the speed of migration calculated solely during the migratory phases with respect to the two RMS subregions. These data are consistent with our previous report showing that endothelial cells synthesize and secrete BDNF, which promotes the entry of neuroblasts into the migratory phase, but does not affect the speed of migration (Snapyan et al., 2009). Although BDNF expression during RMS development has not been explored yet, it is tempting to speculate that the same mechanism also operates during early development. In the adult forebrain, the expression of BDNF by endothelial cells mostly occurs in the RMS (Snapyan et al., 2009). The mechanisms involved in this specific pattern of expression are unknown. However, in the adult songbird brain, the testosterone-mediated increase in VEGF levels induces the expression of BDNF by endothelial cells (Louissaint et al., 2002). Our results showed that, during postnatal development, VEGF is expressed by astrocytes in the SVZ-RMS but not in the cortex or cerebellum. It is therefore conceivable that, in addition to creating an angiogenesis-permissive environment, this spatially restricted and temporally regulated expression of VEGF by the RMS astrocytes also induces the expression of BDNF in the endothelial cells of the migratory stream.

VEGF plays a role in neuronal migration during development (Schwarz et al., 2004) and after injury (Wang et al., 2007). Recently, it has been demonstrated also that Purkinje cell-derived VEGF guides granule cell migration in the cerebellum (Ruiz de Almodovar et al., 2010). However, the role of VEGF in neuroblast migration in the adult RMS remains controversial (Wittko et al., 2009; Licht et al., 2010). Wittko et al. (2009) have demonstrated that VEGFR-1 (Flt-1) is expressed exclusively by astrocytes and that VEGFR-1 signaling-deficient mice have a larger number of neuroblasts in the OB because of the increased levels of VEGF. The authors suggested that higher VEGF levels induce the phosphorylation of VEGFR-2 in neuronal precursors and promote neuroblast migration (Wittko et al., 2009). In contrast, Licht et al. (2010), who specifically blocked VEGF signaling in adulthood by conditionally sequestering VEGF, did not observe a reduction in the number of SVZ-born neurons entering the OB. Interestingly, however, sequestering VEGF during early developmental stages induces the collapse of the RMS vascular network and, as a result, abolishes neuroblast migration along the migratory pathway (Licht et al., 2010). This is in agreement with the results we report here. We showed that VEGF downregulation in vivo during early developmental stages affects vasculature formation and growth in the RMS and, as a result, the migration of neuronal precursors. We were unable to detect a direct role for VEGF in neuroblast migration using videoimaging experiments to track neuronal displacement in real time. Our results indicated that VEGF plays an indirect role in neuronal migration by regulating BV formation and growth. Importantly, we have shown that altered vasculature scaffold in the RMS decreases the number of newborn neurons arriving to the $\mathrm{OB}$. This decrease in the number of newborn neurons in the $\mathrm{OB}$ was accompanied by the accumulation of neuronal precursors in the RMS. We did not observe, however, immigration of neuroblasts from the RMS to the adjacent brain regions. Our preliminary data suggest that despite dramatic alteration in the vasculature scaffold, some vasculature-derived molecular cues remain expressed by the endothelial cells that may retain neuroblasts in the RMS. Thus, as in adult RMS, in the developing migratory stream the vasculature does not only serve as a physical scaffold for neuroblast migration but also may provide molecular cues required for faithful migration.

Altogether, our findings shed light on the impact of astroglial network remodeling on the development and reorganization of the RMS vasculature, an important migration-promoting scaffold in the developing and adult migratory streams.

\section{References}

Alves JA, Barone P, Engelender S, Fróes MM, Menezes JR (2002) Initial stages of radial glia astrocytic transformation in the early postnatal anterior subventricular zone. J Neurobiol 52:251-265.

Anton ES, Ghashghaei HT, Weber JL, McCann C, Fischer TM, Cheung ID, Gassmann M, Messing A, Klein R, Schwab MH, Lloyd KC, Lai C (2004) Receptor tyrosine kinase ErbB4 modulates neuroblast migration and placement in the adult forebrain. Nat Neurosci 7:1319-1328.

Bolteus AJ, Bordey A (2004) GABA release and uptake regulate neuronal precursor migration in the postnatal subventricular zone. J Neurosci 24:7623-7631.

Chiaramello S, Dalmasso G, Bezin L, Marcel D, Jourdan F, Peretto P, Fasolo A, De Marchis S (2007) BDNF/ TrkB interaction regulates migration of SVZ precursor cells via PI3-K and MAP-K signalling pathways. Eur J Neurosci 26:1780-1790.

Colín-Castelán D, Phillips-Farfán BV, Gutiérrez-Ospina G, Fuentes-Farias AL, Báez-Saldaña A, Padilla-Cortés P, Meléndez-Herrera E (2011) EphB4 is developmentally and differentially regulated in blood vessels throughout the forebrain neurogenic niche in the mouse brain: implications for vascular remodeling. Brain Res 1383:90-98.

Conover JC, Doetsch F, Garcia-Verdugo JM, Gale NW, Yancopoulos GD, Alvarez-Buylla A (2000) Disruption of Eph/ephrin signaling affects migration and proliferation in the adult subventricular zone. Nat Neurosci 3:1091-1097.

Cremer H, Lange R, Christoph A, Plomann M, Vopper G, Roes J, Brown R, Baldwin S, Kraemer P, Scheff S, Barthels D, Rajewsky K, Wille W (1994) Inactivation of the N-CAM gene in mice results in size reduction of the olfactory bulb and deficits in spatial learning. Nature 367:455-459.

Grunewald M, Avraham I, Dor Y, Bachar-Lustig E, Itin A, Jung S, Chimenti S, Landsman L, Abramovitch R, Keshet E (2006) VEGF-induced adult neovascularization: recruitment, retention, and role of accessory cells. Cell 124:175-189.

Hu H, Tomasiewicz H, Magnuson T, Rutishauser U (1996) The role of polysialic acid in migration of olfactory bulb interneuron precursors in the subventricular zone. Neuron 16:735-743.

Kaneko N, Marín O, Koike M, Hirota Y, Uchiyama Y, Wu JY, Lu Q, TessierLavigne M, Alvarez-Buylla A, Okano H, Rubenstein JL, Sawamoto K (2011) New neurons clear the path of astrocytic processes for their rapid migration in the adult brain. Neuron 67:213-223.

Kriegstein A, Alvarez-Buylla A (2009) The glial nature of embryonic and adult neural stem cells. Annu Rev Neurosci 32:149-184.

Law AK, Pencea V, Buck CR, Luskin MB (1999) Neurogenesis and neuronal migration in the neonatal rat forebrain anterior subventricular zone do not require GFAP-positive astrocytes. Dev Biol 216:622-634.

Lee MY, Ju WK, Cha JH, Son BC, Chun MH, Kang JK, Park CK (1999) Expression of vascular endothelial growth factor mRNA following transient forebrain ischemia in rats. Neurosci Lett 265:107-110.

Licht T, Eavri R, Goshen I, Shlomai Y, Mizrahi A, Keshet E (2010) VEGF is required for dendritogenesis of newly born olfactory bulb interneurons. Development 137:261-271.

Lledo PM, Saghatelyan A (2005) Integrating new neurons into the adult olfactory bulb: joining the network, life-death decisions, and the effects of sensory experience. Trends Neurosci 28:248-254. 
Lois C, Alvarez-Buylla A (1994) Long-distance neuronal migration in the adult mammalian brain. Science 264:1145-1148.

Louissaint A Jr, Rao S, Leventhal C, Goldman SA (2002) Coordinated interaction of neurogenesis and angiogenesis in the adult songbird brain. Neuron 34:945-960.

Murase S, Horwitz AF (2002) Deleted in colorectal carcinoma and differentially expressed integrins mediate the directional migration of neural precursors in the rostral migratory stream. J Neurosci 22:3568-3579.

Nguyen-Ba-Charvet KT, Picard-Riera N, Tessier-Lavigne M, Baron-Van Evercooren A, Sotelo C, Chédotal A (2004) Multiple roles for slits in the control of cell migration in the rostral migratory stream. J Neurosci 24:1497-1506.

Ogunshola OO, Antic A, Donoghue MJ, Fan SY, Kim H, Stewart WB, Madri JA, Ment LR (2002) Paracrine and autocrine functions of neuronal vascular endothelial growth factor (VEGF) in the central nervous system. J Biol Chem 277:11410-11415.

Ono M, Yanagawa Y, Koyano K (2005) GABAergic neurons in inferior colliculus of the GAD67-GFP knock-in mouse: electrophysiological and morphological properties. Neurosci Res 51:475-492.

Paratcha G, Ibáñez CF, Ledda F (2006) GDNF is a chemoattractant factor for neuronal precursor cells in the rostral migratory stream. Mol Cell Neurosci 31:505-514.

Pencea V, Luskin MB (2003) Prenatal development of the rodent rostral migratory stream. J Comp Neurol 463:402-418.

Peretto P, Giachino C, Aimar P, Fasolo A, Bonfanti L (2005) Chain formation and glial tube assembly in the shift from neonatal to adult subventricular zone of the rodent forebrain. J Comp Neurol 487:407-427.

Robinson CJ, Stringer SE (2001) The splice variants of vascular endothelial growth factor (VEGF) and their receptors. J Cell Sci 114:853-865.

Ruiz de Almodovar C, Coulon C, Salin PA, Knevels E, Chounlamountri N, Poesen K, Hermans K, Lambrechts D, Van Geyte K, Dhondt J, Dresselaers T, Renaud J, Aragones J, Zacchigna S, Geudens I, Gall D, Stroobants S, Mutin M, Dassonville K, Storkebaum E, et al. (2010) Matrix-binding vascular endothelial growth factor (VEGF) isoforms guide granule cell migration in the cerebellum via VEGF receptor Flk1. J Neurosci 30:15052-15066.

Saghatelyan A, de Chevigny A, Schachner M, Lledo PM (2004) Tenascin-R mediates activity-dependent recruitment of neuroblasts in the adult mouse forebrain. Nat Neurosci 7:347-356.

Schwarz Q, Gu C, Fujisawa H, Sabelko K, Gertsenstein M, Nagy A, Taniguchi M, Kolodkin AL, Ginty DD, Shima DT, Ruhrberg C (2004) Vascular endothelial growth factor controls neuronal migration and cooperates with Sema3A to pattern distinct compartments of the facial nerve. Genes Dev 18:2822-2834.

Sinor AD, Irvin SM, Cobbs CS, Chen J, Graham SH, Greenberg DA (1998) Hypoxic induction of vascular endothelial growth factor (VEGF) protein in astroglial cultures. Brain Res 812:289-291.

Snapyan M, Lemasson M, Brill MS, Blais M, Massouh M, Ninkovic J, Gravel C, Berthod F, Götz M, Barker PA, Parent A, Saghatelyan A (2009) Vasculature guides migrating neuronal precursors in the adult mammalian forebrain via brain-derived neurotrophic factor signaling. J Neurosci 29:4172-4188.

Stone J, Itin A, Alon T, Pe'er J, Gnessin H, Chan-Ling T, Keshet E (1995) Development of retinal vasculature is mediated by hypoxia-induced vascular endothelial growth factor (VEGF) expression by neuroglia. J Neurosci 15:4738-4747.

Tammela T, Enholm B, Alitalo K, Paavonen K (2005) The biology of vascular endothelial growth factors. Cardiovasc Res 65:550-563.

Wang Y, Jin K, Mao XO, Xie L, Banwait S, Marti HH, Greenberg DA (2007) VEGF-overexpressing transgenic mice show enhanced post-ischemic neurogenesis and neuromigration. J Neurosci Res 85:740-747.

Whitman MC, Fan W, Rela L, Rodriguez-Gil DJ, Greer CA (2009) Blood vessels form a migratory scaffold in the rostral migratory stream. J Comp Neurol 516:94-104.

Wittko IM, Schänzer A, Kuzmichev A, Schneider FT, Shibuya M, Raab S, Plate KH (2009) VEGFR-1 regulates adult olfactory bulb neurogenesis and migration of neural progenitors in the rostral migratory stream in vivo. J Neurosci 29:8704-8714. 